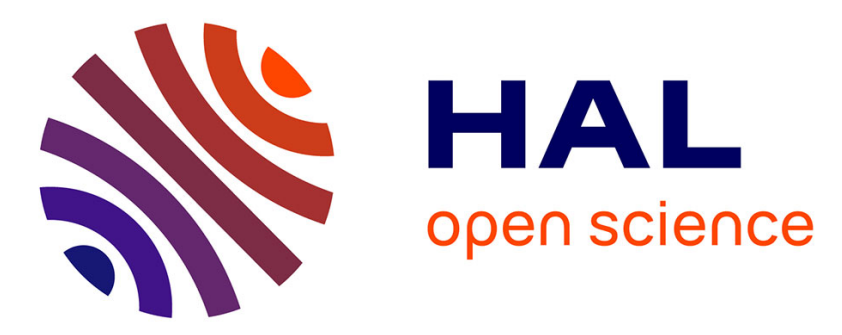

\title{
Bone fibrillogenesis and mineralization: Quantitative analysis and implications for tissue elasticity
}

Jenny Vuong, Christian Hellmich

\section{To cite this version:}

Jenny Vuong, Christian Hellmich. Bone fibrillogenesis and mineralization: Quantitative analysis and implications for tissue elasticity. Journal of Theoretical Biology, 2011, 287, pp.115. 10.1016/j.jtbi.2011.07.028 . hal-00730277

\section{HAL Id: hal-00730277 \\ https://hal.science/hal-00730277}

Submitted on 9 Sep 2012

HAL is a multi-disciplinary open access archive for the deposit and dissemination of scientific research documents, whether they are published or not. The documents may come from teaching and research institutions in France or abroad, or from public or private research centers.
L'archive ouverte pluridisciplinaire HAL, est destinée au dépôt et à la diffusion de documents scientifiques de niveau recherche, publiés ou non, émanant des établissements d'enseignement et de recherche français ou étrangers, des laboratoires publics ou privés. 


\section{Author's Accepted Manuscript}

Bone fibrillogenesis and mineralization: Quantitative analysis and implications for tissue elasticity

Jenny Vuong, Christian Hellmich

PII: S0022-5193(11)00378-X

DOI: doi:10.1016/j.jtbi.2011.07.028

Reference: YJTBI 6569

To appear in: $\quad$ Journal of Theoretical Biology

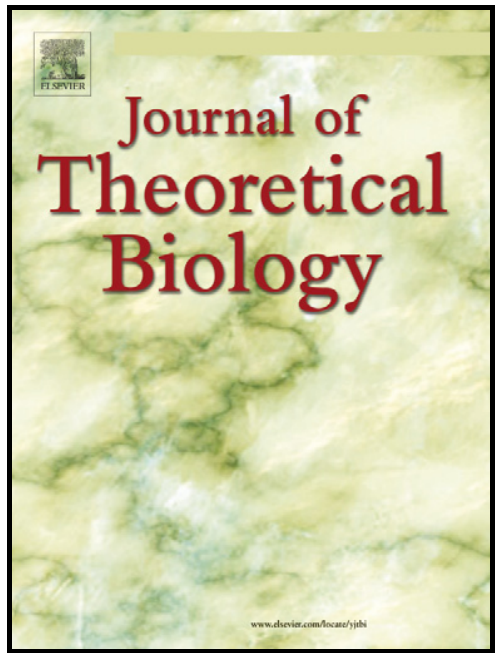

www.elsevier.com/locate/yjtbi

Received date: $\quad 25$ January 2011

Revised date: $\quad 4$ July 2011

Accepted date: $\quad 27$ July 2011

Cite this article as: Jenny Vuong and Christian Hellmich, Bone fibrillogenesis and mineralization: Quantitative analysis and implications for tissue elasticity, Journal of Theoretical Biology, doi:10.1016/j.jtbi.2011.07.028

This is a PDF file of an unedited manuscript that has been accepted for publication. As a service to our customers we are providing this early version of the manuscript. The manuscript will undergo copyediting, typesetting, and review of the resulting galley proof before it is published in its final citable form. Please note that during the production process errors may be discovered which could affect the content, and all legal disclaimers that apply to the journal pertain. 


\title{
Bone fibrillogenesis and mineralization: Quantitative analysis and implications for tissue elasticity
}

\author{
Jenny Vuong*, Christian Hellmich** \\ Institute for Mechanics of Materials and Structures, Vienna University of Technology (TU Wien), A-1040 \\ Wien (Vienna), Austria
}

\begin{abstract}
Data from bone drying, demineralization, and deorganification tests, collected over a time span of more than eighty years, evidence a myriad of different chemical compositions of different bone materials. However, careful analysis of the data, as to extract the chemical concentrations of hydroxyapatite, of water, and of organic material (mainly collagen) in the extracellular bone matrix, reveals an astonishing fact: it appears that there exists a unique bilinear relationship between organic concentration and mineral concentration, across different species, organs, and age groups, from early childhood to OLD AGE: During organ growth, the mineral concentration increases linearly with the organic concentration (which increases during fibrillogenesis), while from adulthood on, further increase of the mineral concentration is accompanied by a decrease in organic concentration. These relationships imply unique mass density-concentration laws for fibrillogenesis and mineralization, which - in combination with micromechanical models - deliver 'universal' mass density-elasticity relationships in extracellular bone matrix - valid across different species, organs, and ages. They turn out as quantitative reflections of the well-instrumented interplay of osteoblasts, osteoclasts, osteocytes, and their precursors, controlling, in a fine-tuned fashion, the chemical genesis and continuous transformation of the extracellular bone matrix. Considerations of the aformentioned rules may strongly affect the potential success of tissue engineering strategies, in particular when translating, via micromechanics, the aformentioned growth and mineralization characteristics into tissue-specific elastic properties.
\end{abstract}

Key words: bone, fibrillogenesis, mineralization, elasticity, continuum micromechanics, Computer Tomography, biomaterials, tissue engineering

*Tel: +43158801 20265; fax: +4315880120299

** Corresponding author. Tel: +43 158801 20220; fax: +43 15880120299

Email addresses: jenny.vuong@ tuwien. ac .at (Jenny Vuong),

christian.hellmichatuwien.ac.at (Christian Hellmich) 


\author{
Nomenclature \\ A \\ $b$ \\ $B$ \\ $\mathbb{C}_{r}$ \\ $\mathbb{C}_{s}$ \\ $\mathbb{C}^{e c}$ \\ $\mathbb{C}^{\text {hom }}$ \\ $\mathbb{C}^{0}$ \\ $C_{i j k l}^{e c}$ \\ $C_{i j k l}^{e x p}$ \\ $C_{i j k l}^{\text {pred }}$ \\ $d_{s}$ \\ D \\ $D_{i j k l}^{e c}$ \\ $\bar{e}$ \\ $e_{s}$ \\ $E_{1}^{e c}$ \\ $E_{3}^{e c}$ \\ $f_{\text {col }}$ \\ $\stackrel{\circ}{f o l}^{\prime}$ \\ $f_{e f}$ \\ $f_{f i b}$ \\ $f_{H A}$ \\ $\breve{f}_{H A}$ \\ $\check{f}_{H A}$ \\ $f_{\mathrm{H}_{2} \mathrm{O}}$ \\ $f_{\text {org }}$ \\ ... regression parameter for function $\mathscr{F}_{\rho_{\text {org }}^{*}}\left(\rho_{H A}^{*}\right)$ \\ ... average (rigid) collagen crosslink length valid for all miner- \\ alized tissues \\ ... regression parameter for function $\mathscr{F}_{\rho_{\text {org }}^{*}}\left(\rho_{H A}^{*}\right)$ \\ .. elasticity tensor of phase $r$ \\ .. elasticity tensor of phase $s$ \\ ... elasticity tensor of extracellular matrix \\ ... homogenized elasticity tensor \\ ... elasticity tensor of matrix in Eshelby's matrix-inclusion \\ problem \\ ... components of elasticity tensor of extracellular matrix \\ ... experimental value for component $i j k l$ of elasticity tensor \\ ... model-predicted value for component $i j k l$ of elasticity tensor \\ ... equatorial diffraction spacing of molecular collagen \\ .. axial macroperiod of staggered assemblies of type I collagen \\ ... components of compliance tensor of extracellular matrix \\ ... mean of relative errors between model-predicted and experi- \\ mental values \\ ... standard deviation of relative errors between model-predicted \\ and experimental values \\ ... Young's modulus in transverse direction, of extracellular \\ bone material \\ ... Young's modulus in axial direction, of extracellular bone ma- \\ terial \\ volume fraction of collagen in extracellular bone matrix \\ volume fraction of (molecular) collagen at the wet collagen \\ level \\ ... volume fraction of the extrafibrillar space \\ ... volume fraction of the fibrillar space \\ ... volume fraction of hydroxyapatite in extracellular matrix \\ ... volume fraction of hydroxyapatite in the fibrillar space \\ ... volume fraction of hydroxyapatite in the extrafibrillar space \\ ... volume fraction of water in extracellular matrix \\ ... volume fraction of organic matter in extracellular matrix
}




\begin{tabular}{|c|c|c|}
\hline$f_{r}$ & $\ldots$ & volume fraction of the phase $r$ \\
\hline$f_{s}$ & $\ldots$ & volume fraction of the phase $s$ \\
\hline$\breve{f}_{\text {wetcol }}$ & $\ldots$ & volume fraction wet collagen in the fibrillar space \\
\hline $\mathscr{F}_{\rho_{\text {org }}^{*}}\left(\rho_{H A}^{*}\right)$ & & bilinear relationship between $\rho_{\text {org }}^{*}$ and $\rho_{H A}^{*}$ \\
\hline $\mathfrak{F}\left(A, B, \rho_{H A}^{*, c r i t}\right)$ & $\cdots$ & $\begin{array}{l}\text { sum of squares of the differences between experimental and } \\
\text { predicted values }\end{array}$ \\
\hline$g$ & $\ldots$ & standard average gravity \\
\hline$G_{12}^{e c}$ & $\ldots$ & $\begin{array}{l}\text { shear modulus in isotropic plane } 1-2 \text {, of extracellular bone } \\
\text { material }\end{array}$ \\
\hline$i$ & $\cdots$ & index (numbering of experimental values) \\
\hline $\mathbb{I}$ & $\ldots$ & fourth-order unity tensor \\
\hline$m_{0}$ & $\ldots$ & mass constant in diffraction-density relation \\
\hline$M_{a i r}$ & $\ldots$ & $\begin{array}{l}\text { mass of (millimeter-sized) wet bone sample in air (with } \\
\text { empty vascular pores) }\end{array}$ \\
\hline$M_{d r y}$ & $\ldots$ & mass of (millimeter-sized) dehydrated bone sample \\
\hline$M_{H A}$ & $\ldots$ & mass of hydroxyapatite in (millimeter-sized) bone sample \\
\hline $\mathrm{M}_{\mathrm{H}_{2} \mathrm{O}}$ & $\cdots$ & mass of water in (millimeter-sized) bone sample \\
\hline$M_{\text {org }}$ & & mass of organic material in (millimeter-sized) bone sample \\
\hline$N$ & & number of experimental values \\
\hline $\mathbb{P}_{r}^{0}$ & & $\begin{array}{l}\text { fourth-order Hill tensor of phase } r \text { being embedded in matrix } \\
\text { with stiffness } \mathbb{C}^{0}\end{array}$ \\
\hline$R^{2}$ & & coefficient of determination \\
\hline$S S_{e r r}$ & & sum of squares of residuals \\
\hline$S S_{t o t}$ & & total sum of squares \\
\hline$v_{f i b}$ & & volume of one rhomboidal fibrillar unit \\
\hline$v_{1}$ & & $\begin{array}{l}\text { velocitiy of acoustic plane wave travelling in transverse di- } \\
\text { rection }\end{array}$ \\
\hline$v_{3}$ & $\cdots$ & velocitiy of acoustic plane wave travelling in axial direction \\
\hline$V^{e c}$ & $\ldots$ & $\begin{array}{l}\text { volume of the extracellular tissue within (millimeter-sized) } \\
\text { bone sample }\end{array}$ \\
\hline$W_{\text {submerged }}$ & $\cdots$ & $\begin{array}{l}\text { weight of (millimeter-sized) bone sample when fully sub- } \\
\text { merged in liquid }\end{array}$ \\
\hline$W F_{a s h}^{d r y}$ & $\ldots$ & weight fraction of ash in dried bone samples \\
\hline$W F_{H A}$ & $\cdots$ & $\begin{array}{l}\text { weight fraction of hydroxyapatite in extracellular bone ma- } \\
\text { trix }\end{array}$ \\
\hline
\end{tabular}




\begin{tabular}{|c|c|c|}
\hline$W F_{\mathrm{H}_{2} \mathrm{O}}$ & $\cdots$ & weight fraction of water in extracellular bone matrix \\
\hline$W F_{\text {org }}$ & $\ldots$ & weight fraction of organic material in extracellular bone matrix \\
\hline$\mu^{e c}$ & $\ldots$ & $\mathrm{X}$-ray attenuation coefficient of the extracellular bone material \\
\hline$\mu_{H A}$ & $\ldots$ & X-ray attenuation coefficient of hydroxyapatite \\
\hline$\mu_{\mathrm{H}_{2} \mathrm{O}}$ & $\cdots$ & X-ray attenuation coefficient of water \\
\hline$\mu_{\text {org }}$ & $\ldots$ & $\mathrm{X}$-ray attenuation coefficient of organic matter \\
\hline$\nu_{12}$ & .. & Poisson's ratio in isotropic plane, of extracellular bone material \\
\hline$\nu_{13}$ & $\ldots$ & Poisson's ratio in axial direction, of extracellular bone material \\
\hline$\rho^{e c}$ & $\ldots$ & mass density of extracellular bone matrix \\
\hline$\rho_{\text {liquid }}$ & $\cdots$ & mass density of liquid \\
\hline$\rho_{H A}$ & $\cdots$ & real mass density of hydroxyapatite \\
\hline$\rho_{\mathrm{H}_{2} \mathrm{O}}$ & $\ldots$ & real mass density of water \\
\hline$\rho_{\text {org }}$ & $\cdots$ & real mass density of organic matter \\
\hline$\rho_{H A}^{*}$ & $\ldots$ & $\begin{array}{l}\text { apparent mass density of hydroxyapatite (mass of hydroxyapatite } \\
\text { per volume of extracellular bone matrix) }\end{array}$ \\
\hline$\rho_{\mathrm{H}_{2} \mathrm{O}}^{*}$ & $\ldots$ & $\begin{array}{l}\text { apparent mass density of water (mass of water per volume of extra- } \\
\text { cellular bone matrix) }\end{array}$ \\
\hline$\rho_{o r g}^{*}$ & $\ldots$ & $\begin{array}{l}\text { apparent mass density of organic matter (mass of organic matter per } \\
\text { volume of extracellular bone matrix) }\end{array}$ \\
\hline$\phi_{H A, e f}$ & $\ldots$ & $\begin{array}{l}\text { relative amount of hydroxyapatite in the extrafibrillar space, with } \\
\text { respect to total amount of hydroxyapatite per volume of extracellu- } \\
\text { lar matrix }\end{array}$ \\
\hline
\end{tabular}




\section{Introduction}

Bone tissue engineering has become a huge field, both in terms of scientific production and medical potential [Cancedda et al., 2007], and ever since the pioneering work of Langer and Vacanti [1993], a great variety of different material systems have been explored for their potential use as bone tissue engineering scaffolds [Karageorgiou and Kaplan, 2005; Perry, 2002; Komlev et al., 2010; Verma et al., 2008]. Quite naturally among all these materials, such based on the actual elementary components of bone, i.e. on hydroxyapatite, collagen and water, may play an important role among all the aformentioned material classes, since one might well expect that such materials might tend to be able to reproduce both the astonishing mechanical properties of bone and the material's biological features [Ficai et al., 2009; Green, 2008; Pramatarova et al., 2005; Roeder, 2008]. But even if the materials' basic components are chosen, the questions regarding their mixing characteristics, i.e. the concentrations of the individual components, remain to be answered. A close inspection of century-long chemical investigations clearly shows that there is a large variety of compositions, ranging all the way from (almost) unmineralized osteoid in early deposition stages, to tissues consisting mainly of hydroxyapatite [Hammett, 1925; Lees et al., 1995]. To somehow simplify this truly complicated matter, one might hope for general inherent 'rules', quantifying natural relationships between constituent concentrations stemming from the sophistically orchestrated activity of biological cells, hormones, and growth factors [Lemaire et al., 2004; Gajjeraman et al., 2007; Filvaroff and Derynck, 1998; Pivonka et al., 2010]. Recalling, FROM [RIEDL, 1978] AND [GOULD AND LEWONTIN, 1979], THAT “THE LIVING WORLD HAPPENS TO BE CROWDED BY UNIVERSAL PATTERNS OF ORGANIZATION WHICH, MOST OBVIOUSLY, FIND NO DIRECT EXPLANATION THROUGH ENVIRONMENTAL CONDITIONS OR ADAPTIVE RADIATION", IT IS THE VERY FOCUS OF THIS PAPER TO CHECK FOR THE EXISTENCE OF SUCH 'UNIVERSAL' RULES IN BONE FIBRILLOGENESIS [WHEN MORE AND MORE ORGANIC MATTER, MOSTLY COLLAGEN, IS ACCUMULATED IN A MATERIAL VOLUME OF EXTRACELLULAR BONE TISSUE [HAMMETT, 1925; PARRY AND CRAIG, 1978]] AND IN BONE MINERALIZATION [WHEN HYDROXYAPATITE PRECIPITATES IN THE EXTRACOLLAGENOUS SPACES OF EXTRACELLULAR BONE TISSUE [HELLMICH AND UlM, 2003; HöHLING, 1969; LANDIS ET AL., 1996]]. Therefore, we aim at quantification of relations between the concentrations of the elementary components within a piece of extracellular bone matrix, being expressed as the masses of these constituents per volume of extraellular bone matrix ("apparent mass densities"). In order to reach this aim, we perform the following steps, as described in the sequel of the paper: We collect a comprehensive data base from the literature, related to tissue mass density and composition measurements from a great variety of tissues, belonging either to the same tissue type at different ages of the organism, or to tissues from different organisms at only one age per organism, or both. Subsequently, we describe how to derive constituent concentrations (i.e. apparent mass densities of mineral, collagen and water) from these experimental data base (Section 2). The results of this derivation, 'universal' rules for bone fibrillogenesis and mineralization, are given thereafter (Section 3 ). Then, the aformentioned bone composition rules are converted, through micromechanics laws, into composition-elasticity relations, which may be used as a prerequi- 
site for biomaterial design (Section 4). Finally, the results are discussed in view of their relation to modern bone biology (SECTION 5), BEFORE GIVING AN OUTLOOK TO USEFUl APPLICATIONS OF OUR FINDINGS (SECTION 6). THROUGHOUt THE MANUSCRIPT, WE USE THE TERM "UNIVERSAL" WITH CAUTION (AND HENCE IN QUOTATION MARKS), DISCUSSING, AT THE SAME TIME, THE VALIDITY RANGES OF THE IDENTIFIED RELATIONS, AS WELL AS THEIR LIMITATIONS.

\section{Evaluation of mass and volume, demineralization and ashing experiments -} mass densities and concentrations

\subsection{Mass and volume measurements - mass densities}

The experimental data of Lees et al. [1979a, 1995, 1983]; Lees [1987, 2003]; Lees and Page [1992]; Biltz and Pellegrino [1969]; Gong et al. [1964a]; Burns [1929]; Hammett [1925] refer to mass density determination according to Archimedes' principle: The mass of samples with a typical size of a few millimeters is measured in air. Hence, the respective value $M_{\text {air }}$ refers to a state of empty vascular and lacunar pores, but with wet extracellular (ec) bone matrix. This mass is related to the volume of the extracellular bone matrix $V^{e c}$, as to arrive at the extracellular tissue mass density

$$
\rho^{e c}=\frac{M_{a i r}}{V^{e c}} .
$$

The important feature of Archimedes' principle lies in the mode of determination of $V^{e c}$. It is determined from $M_{a i r}$, together with the weight $W_{\text {submerged }}$ of the sample when submerged into a liquid with mass density $\rho_{\text {liquid }}$, according to

$$
V^{e c}=\frac{M_{\text {air }}-\frac{W_{\text {submerged }}}{g}}{\rho_{\text {liquid }}},
$$

with $g$ as the standard average gravity, $g=9.81 \mathrm{~m} / \mathrm{s}^{2}$. Eq. (2) is valid once the pressure in the vascular and lacunar pores follows the hydrostatic pressure distribution in the container where the sample is submerged. Given the characteristic size of vascular and lacunar pores, this requirement is standardly fulfilled. Still, care has to be taken that no air bubbles are entrapped when submerging the samples [Gong et al., 1964a]. Extracellular bone tissue mass densities as determined from Eqs. (1) and (2) have been documented by Lees et al. [1979a, 1983]; Lees [1987]; Lees and Page [1992]; Lees et al. [1995]; Lees [2003]; Biltz and Pellegrino [1969]; Broz et al. [1995]; Gong et al. [1964a]; Burns [1929]; Hammett [1925], see Tables 1-5.

2.2. Dehydration-demineralization tests - Concentrations of hydroxyapatite, organics, and water

According to the protocols of Lees et al. [1979a, 1983]; Lees [1987]; Lees and Page [1992], Lees et al. [1995]; Lees [2003], bone samples are dried in a vacuum dessicator at room temperature, until a constant mass is observed (typically after 7 days). This 
mass is the mass of the dehydrated extracellular bone tissue, $M_{d r y}$. The difference between the wet tissue mass in air, $M_{a i r}$, and the dehydrated tissue mass, $M_{d r y}$, equals the mass of water contained in the extracellular matrix,

$$
M_{H_{2} O}=M_{a i r}-M_{d r y}
$$

The water content is typically given in terms of the weight fraction of water,

$$
W F_{H_{2} O}=\frac{M_{H_{2} O}}{M_{a i r}},
$$

or in terms of the apparent mass density

$$
\rho_{H_{2} O}^{*}=\frac{M_{H_{2} O}}{V^{e c}}
$$

which is proportional to the chemical concentration of water, with the molar mass of water as the proportionality factor. Next, the samples are re-hydrated and then demineralized in a $0.5 \mathrm{M}$ EDTA solution at $\mathrm{pH} 7.5$, until no calcium is detected anymore by an atomic absorption spectrometer. After drying such a demineralized sample in vaccum, one is left with the organic mass contained in the tissue, $M_{\text {org }}$. The respective organic content is typically given in terms of the organic weight fraction

$$
W F_{\text {org }}=\frac{M_{\text {org }}}{M_{\text {air }}}
$$

or in terms of the apparent mass density

$$
\rho_{\text {org }}^{*}=\frac{M_{\text {org }}}{V^{e c}},
$$

which is proportional to the chemical concentration of organic matter within the extracellular bone matrix, with the molar mass of the organic material as the proportionality factor. Finally, knowledge of masses and concentrations of both organic and water gives access to the hydroxyapatite masses, weight fractions, and apparent mass densities, as

$$
\begin{gathered}
M_{H A}=M_{a i r}-M_{o r g}-M_{H_{2} O}, \\
W F_{H A}=\frac{M_{H A}}{M_{a i r}},
\end{gathered}
$$




$$
\rho_{H A}^{*}=\frac{M_{H A}}{V^{e c}},
$$

and the subsequent relations follow from Eqs. (3)-(10)

$$
\begin{gathered}
W F_{H A}+W F_{o r g}+W F_{H_{2} O}=1, \\
\rho_{H A}^{*}+\rho_{o r g}^{*}+\rho_{H_{2} O}^{*}=\rho^{e c},
\end{gathered}
$$

with the apparent mass densities calculated via

$$
\begin{gathered}
\rho_{o r g}^{*}=W F_{o r g} \times \rho^{e c}, \\
\rho_{H A}^{*}=W F_{H A} \times \rho^{e c}, \\
\rho_{H_{2} O}^{*}=W F_{H_{2} O} \times \rho^{e c} .
\end{gathered}
$$

Such weight fractions have been documented by Lees et al. [1979a, 1983]; Lees [1987]; Lees and Page [1992]; Lees et al. [1995]; Lees [2003], and Eqs. (13) to (15) are used to compute the apparent mass densities, as given in Tables 1 and 2. The extracellular mass densities $\rho^{e c}$ are not explicitly given in [Lees, 2003]. In this case, the weight fractions provide access to $\rho^{e c}$, via

$$
\begin{aligned}
\rho^{e c} & =\left(\frac{W F_{\text {org }}}{\rho_{\text {org }}}+\frac{W F_{H A}}{\rho_{H A}}+\frac{W F_{H_{2} O}}{\rho_{H_{2} O}}\right)^{-1} \\
& =\left(\frac{W F_{\text {org }}}{\rho_{\text {org }}}+\frac{W F_{H A}}{\rho_{H A}}+\frac{1-W F_{\text {org }}-W F_{H A}}{\rho_{H_{2} O}}\right)^{-1}
\end{aligned}
$$

with the real mass densities of water, of organics, and of hydroxyapatite amounting to $\rho_{\mathrm{H}_{2} \mathrm{O}}=1 \mathrm{~g} / \mathrm{cm}^{3}, \rho_{\text {org }}=1.41 \mathrm{~g} / \mathrm{cm}^{3}$ [Lees, 1987], and $\rho_{\mathrm{HA}}=3 \mathrm{~g} / \mathrm{cm}^{3}$ [Gong et al., 1964b; Lees, 1987; Hellmich, 2005]. Corresponding values are documented in Table 3. 
Table 1: Bone compositions from dehydration-demineralization experiments of Lees et al. [1979a]; Lees and Page [1992]

\begin{tabular}{|c|c|c|c|c|c|c|c|}
\hline Tissue & $\rho^{e c}$ & $W F_{H A}$ & $W F_{\text {org }}$ & $W F_{H_{2}} \mathrm{O}$ & $\rho_{H A}^{*}$ & $\rho_{\text {org }}^{*}$ & $\rho_{\mathrm{H}_{2} \mathrm{O}}^{*}$ \\
\hline & $\begin{array}{l}{\left[\mathrm{g} / \mathrm{cm}^{3}\right]} \\
\text { given }\end{array}$ & $\begin{array}{l}- \\
\text { given } \\
\end{array}$ & $\begin{array}{l}- \\
\text { given }\end{array}$ & $\begin{array}{l}- \\
\text { given }\end{array}$ & $\begin{array}{l}{\left[\mathrm{g} / \mathrm{cm}^{3}\right]} \\
\text { Eq. }(14)\end{array}$ & $\begin{array}{l}{\left[\mathrm{g} / \mathrm{cm}^{3}\right]} \\
\text { Eq. }(13)\end{array}$ & $\begin{array}{l}{\left[\mathrm{g} / \mathrm{cm}^{3}\right]} \\
\text { Eq. }(15) \\
\end{array}$ \\
\hline Bovine tibia $^{\mathrm{a}}$ & 2.060 & 0.66 & 0.22 & 0.12 & 1.355 & 0.451 & 0.253 \\
\hline Bovine tibia $^{\mathrm{a}}$ & 2.050 & 0.66 & 0.22 & 0.13 & 1.345 & 0.449 & 0.258 \\
\hline Bovine tibia $^{\mathrm{a}}$ & 2.020 & 0.62 & 0.24 & 0.14 & 1.254 & 0.483 & 0.283 \\
\hline Bovine tibia $^{\mathrm{a}}$ & 2.020 & 0.63 & 0.23 & 0.14 & 1.267 & 0.469 & 0.283 \\
\hline Bovine tibia $^{\mathrm{a}}$ & 2.000 & 0.64 & 0.23 & 0.13 & 1.286 & 0.454 & 0.258 \\
\hline Bovine tibia $^{\mathrm{a}}$ & 2.050 & 0.64 & 0.23 & 0.13 & 1.318 & 0.471 & 0.260 \\
\hline Bovine tibia $^{\mathrm{a}}$ & 2.100 & 0.67 & 0.21 & 0.12 & 1.409 & 0.443 & 0.248 \\
\hline Bovine tibia $^{\mathrm{a}}$ & 2.080 & 0.66 & 0.22 & 0.12 & 1.381 & 0.449 & 0.250 \\
\hline Bovine tibia $^{a}$ & 2.020 & 0.66 & 0.22 & 0.12 & 1.329 & 0.442 & 0.248 \\
\hline Bovine tibia $^{\mathrm{a}}$ & 1.990 & 0.66 & 0.22 & 0.13 & 1.305 & 0.436 & 0.251 \\
\hline Bovine tibia $^{\mathrm{a}}$ & 1.950 & 0.64 & 0.23 & 0.13 & 1.248 & 0.445 & 0.255 \\
\hline Bovine tibia $^{\mathrm{a}}$ & 2.010 & 0.66 & 0.22 & 0.12 & 1.325 & 0.438 & 0.247 \\
\hline Bovine tibia $^{\mathrm{a}}$ & 2.040 & 0.64 & 0.24 & 0.12 & 1.302 & 0.494 & 0.247 \\
\hline Bovine tibia $^{\mathrm{a}}$ & 2.050 & 0.70 & 0.21 & 0.12 & 1.433 & 0.430 & 0.238 \\
\hline Bovine tibia $^{\mathrm{a}}$ & 2.120 & 0.66 & 0.21 & 0.12 & 1.401 & 0.456 & 0.261 \\
\hline Bovine tibia $^{\mathrm{a}}$ & 2.080 & 0.66 & 0.22 & 0.12 & 1.379 & 0.460 & 0.241 \\
\hline Bovine tibia $^{\mathrm{a}}$ & 2.100 & 0.65 & 0.22 & 0.13 & 1.359 & 0.470 & 0.271 \\
\hline Bovine tibia $^{\mathrm{a}}$ & 1.980 & 0.65 & 0.22 & 0.13 & 1.295 & 0.430 & 0.253 \\
\hline Bovine tibia $^{\mathrm{a}}$ & 2.050 & 0.64 & 0.23 & 0.13 & 1.320 & 0.465 & 0.264 \\
\hline Bovine tibia $^{\mathrm{a}}$ & 2.110 & 0.65 & 0.23 & 0.12 & 1.369 & 0.483 & 0.257 \\
\hline Bovine tibia $^{\mathrm{a}}$ & 2.030 & 0.64 & 0.21 & 0.12 & 1.295 & 0.432 & 0.250 \\
\hline Bovine tibia $^{\mathrm{a}}$ & 2.060 & 0.70 & 0.18 & 0.12 & 1.440 & 0.379 & 0.241 \\
\hline Mineralized turkey leg tendon ${ }^{\mathrm{b}}$ & 1.332 & 0.29 & 0.25 & 0.46 & 0.380 & 0.333 & 0.619 \\
\hline Mineralized turkey leg tendon ${ }^{\mathrm{b}}$ & 1.498 & 0.45 & 0.24 & 0.32 & 0.667 & 0.358 & 0.473 \\
\hline Mineralized turkey leg tendon ${ }^{\mathrm{b}}$ & 1.498 & 0.41 & 0.22 & 0.37 & 0.614 & 0.325 & 0.560 \\
\hline Mineralized turkey leg tendon ${ }^{\mathrm{b}}$ & 1.507 & 0.44 & 0.22 & 0.35 & 0.658 & 0.326 & 0.522 \\
\hline Mineralized turkey leg tendon ${ }^{\mathrm{b}}$ & 1.520 & 0.45 & 0.24 & 0.31 & 0.690 & 0.363 & 0.468 \\
\hline Mineralized turkey leg tendon ${ }^{b}$ & 1.523 & 0.44 & 0.22 & 0.34 & 0.666 & 0.334 & 0.523 \\
\hline Mineralized turkey leg tendon ${ }^{\mathrm{b}}$ & 1.524 & 0.40 & 0.24 & 0.36 & 0.603 & 0.372 & 0.549 \\
\hline Mineralized turkey leg tendon ${ }^{\mathrm{b}}$ & 1.533 & 0.44 & 0.22 & 0.34 & 0.678 & 0.341 & 0.514 \\
\hline Mineralized turkey leg tendon ${ }^{\mathrm{b}}$ & 1.541 & 0.46 & 0.24 & 0.30 & 0.707 & 0.376 & 0.457 \\
\hline Mineralized turkey leg tendon ${ }^{\mathrm{b}}$ & 1.577 & 0.47 & 0.23 & 0.30 & 0.746 & 0.359 & 0.472 \\
\hline Mineralized turkey leg tendon ${ }^{\mathrm{b}}$ & 1.581 & 0.46 & 0.22 & 0.32 & 0.730 & 0.343 & 0.508 \\
\hline Mineralized turkey leg tendon ${ }^{\mathrm{b}}$ & 1.589 & 0.48 & 0.23 & 0.30 & 0.756 & 0.362 & 0.471 \\
\hline Mineralized turkey leg tendon ${ }^{\mathrm{b}}$ & 1.597 & 0.49 & 0.23 & 0.28 & 0.777 & 0.368 & 0.452 \\
\hline Mineralized turkey leg tendon ${ }^{\mathrm{b}}$ & 1.610 & 0.46 & 0.23 & 0.31 & 0.739 & 0.371 & 0.500 \\
\hline Mineralized turkey leg tendon ${ }^{b}$ & 1.614 & 0.49 & 0.24 & 0.26 & 0.799 & 0.394 & 0.421 \\
\hline Mineralized turkey leg tendon ${ }^{\mathrm{b}}$ & 1.619 & 0.50 & 0.23 & 0.27 & 0.810 & 0.369 & 0.440 \\
\hline Mineralized turkey leg tendon ${ }^{\mathrm{b}}$ & 1.643 & 0.51 & 0.23 & 0.27 & 0.831 & 0.374 & 0.438 \\
\hline
\end{tabular}


Table 2: Bone compositions from dehydration-demineralization experiments of Lees et al. [1995]

\begin{tabular}{|c|c|c|c|c|c|c|c|}
\hline Tissue & $\rho^{e c}$ & $W F_{H A}$ & $W F_{\text {org }}$ & $W F_{\mathrm{H}_{2} \mathrm{O}}$ & $\rho_{H A}^{*}$ & $\rho_{\text {org }}^{*}$ & $\rho_{\mathrm{H}_{2} \mathrm{O}}^{*}$ \\
\hline & $\begin{array}{l}{\left[\mathrm{g} / \mathrm{cm}^{3}\right]} \\
\text { given }\end{array}$ & given & given & given & $\begin{array}{l}{\left[\mathrm{g} / \mathrm{cm}^{3}\right]} \\
\text { Eq. (14) }\end{array}$ & $\begin{array}{l}{\left[\mathrm{g} / \mathrm{cm}^{3}\right]} \\
\text { Eq. (13) }\end{array}$ & $\begin{array}{l}{\left[\mathrm{gg} / \mathrm{cm}^{3}\right]} \\
\text { Eq. }(15)\end{array}$ \\
\hline Whale malleus $\mathrm{a}$ & 2.490 & 0.86 & 0.10 & 0.04 & 2.141 & 0.249 & 0.100 \\
\hline Whale malleus a & 2.450 & 0.80 & 0.13 & 0.07 & 1.960 & 0.319 & 0.172 \\
\hline Whale incus ${ }^{a}$ & 2.500 & 0.86 & 0.09 & 0.05 & 2.150 & 0.225 & 0.125 \\
\hline Whale stapes $\mathrm{a}$ & 2.420 & 0.81 & 0.13 & 0.06 & 1.960 & 0.315 & 0.145 \\
\hline Whale stapes ${ }^{a}$ & 2.360 & 0.80 & 0.14 & 0.06 & 1.888 & 0.330 & 0.142 \\
\hline Whale periotic ${ }^{\mathrm{a}}$ & 2.400 & 0.81 & 0.13 & 0.07 & 1.944 & 0.312 & 0.168 \\
\hline Whale periotic ${ }^{\mathrm{a}}$ & 2.480 & 0.83 & 0.11 & 0.06 & 2.058 & 0.273 & 0.149 \\
\hline Whale periotic ${ }^{\mathrm{a}}$ & 2.520 & 0.85 & 0.10 & 0.05 & 2.142 & 0.252 & 0.126 \\
\hline Whale periotic ${ }^{\mathrm{a}}$ & 2.520 & 0.85 & 0.10 & 0.05 & 2.142 & 0.252 & 0.126 \\
\hline Whale periotic ${ }^{\mathrm{a}}$ & 2.580 & 0.87 & 0.09 & 0.04 & 2.245 & 0.232 & 0.103 \\
\hline Whale t. bulla $\mathrm{a}^{\mathrm{a}}$ & 2.480 & 0.85 & 0.10 & 0.05 & 2.108 & 0.248 & 0.124 \\
\hline
\end{tabular}

Table 3: Bone compositions from dehydration-demineralization experiments of Lees [2003]

\begin{tabular}{|c|c|c|c|c|c|c|c|}
\hline Tissue & $W F_{H A}$ & $W F_{\text {org }}$ & $W F_{H_{2}} \mathrm{O}$ & $\rho^{e c}$ & $\rho_{H A}^{*}$ & $\rho_{\text {org }}^{*}$ & $\rho_{\mathrm{H}_{2} \mathrm{O}}^{*}$ \\
\hline & $\begin{array}{l}- \\
\text { given } \\
\end{array}$ & & $\begin{array}{l}- \\
\text { given } \\
\end{array}$ & $\begin{array}{l}{\left[\mathrm{g} / \mathrm{cm}^{3}\right]} \\
\text { Eq. (16) }\end{array}$ & $\begin{array}{l}{\left[\mathrm{g} / \mathrm{cm}^{3}\right]} \\
\text { Eq. (14) }\end{array}$ & $\begin{array}{l}{\left[\mathrm{g} / \mathrm{cm}^{3}\right]} \\
\text { Eq. (13) }\end{array}$ & $\begin{array}{l}{\left[\mathrm{g} / \mathrm{cm}^{3}\right]} \\
\text { Eq. (15) }\end{array}$ \\
\hline Horse metacarpal & 0.54 & 0.25 & 0.20 & 1.784 & 0.981 & 0.446 & 0.357 \\
\hline Horse metacarpal & 0.53 & 0.26 & 0.17 & 1.837 & 1.047 & 0.478 & 0.312 \\
\hline Horse metacarpal & 0.54 & 0.26 & 0.19 & 1.793 & 0.986 & 0.466 & 0.341 \\
\hline Horse metacarpal & 0.63 & 0.28 & 0.18 & 1.790 & 0.967 & 0.501 & 0.322 \\
\hline Horse metacarpal & 0.62 & 0.26 & 0.12 & 1.957 & 1.213 & 0.509 & 0.235 \\
\hline Horse metacarpal & 0.62 & 0.27 & 0.11 & 1.968 & 1.220 & 0.531 & 0.216 \\
\hline Horse metacarpal & 0.64 & 0.26 & 0.12 & 1.957 & 1.213 & 0.509 & 0.235 \\
\hline Horse metacarpal & 0.62 & 0.26 & 0.13 & 1.932 & 1.178 & 0.502 & 0.251 \\
\hline Horse metacarpal & 0.66 & 0.25 & 0.13 & 1.946 & 1.206 & 0.486 & 0.253 \\
\hline Horse metacarpal & 0.63 & 0.23 & 0.23 & 1.745 & 0.942 & 0.401 & 0.401 \\
\hline Horse metacarpal & 0.54 & 0.24 & 0.23 & 1.733 & 0.919 & 0.416 & 0.399 \\
\hline Horse metacarpal & 0.53 & 0.27 & 0.19 & 1.781 & 0.962 & 0.481 & 0.338 \\
\hline Horse metacarpal & 0.54 & 0.22 & 0.15 & 1.938 & 1.221 & 0.426 & 0.291 \\
\hline Horse metacarpal & 0.63 & 0.25 & 0.13 & 1.946 & 1.206 & 0.486 & 0.253 \\
\hline Horse metacarpal & 0.62 & 0.26 & 0.12 & 1.957 & 1.213 & 0.509 & 0.235 \\
\hline Horse metacarpal & 0.62 & 0.23 & 0.13 & 1.975 & 1.264 & 0.454 & 0.257 \\
\hline Horse metacarpal & 0.64 & 0.26 & 0.12 & 1.957 & 1.213 & 0.509 & 0.235 \\
\hline Horse metacarpal & 0.62 & 0.23 & 0.12 & 1.988 & 1.312 & 0.457 & 0.239 \\
\hline Horse metacarpal & 0.66 & 0.24 & 0.13 & 1.960 & 1.235 & 0.470 & 0.255 \\
\hline
\end{tabular}




\subsection{Dehydration-deorganification tests - Concentrations of hydroxyapatite, organics, and water}

According to the protocol of Gong et al. [1964a], bone samples with original weight of $M_{a i r}$ are dried at 80 for 72 hours, after which they exhibit mass $M_{d r y}$. Through Eqs. (3)-(5), these masses give access to the water concentration in the extracellular bone matrices. Next, the samples are freed from fat and other organic material, using, in a soxhlet apparatus, a mixture of $80 \%$ ethyl ether and $20 \%$ ethyl alcohol, as well as an $80 \%$ aqueous solution of ethylene diamine, respectively.

After drying such a de-organified sample at 80 until a constant weight is attained, one is left with the hydroxyapatite mass contained in the tissue, $M_{H A}$. Through Eqs. (9)-(10), this mass gives access to the hydroxyapatite concentrations in the extracellular bone matrices. Finally, when knowing $M_{H A}, M_{a i r}$, and $M_{H_{2} O}$, Eq. (8), together with Eq. (6) and (7), delivers the organic concentration. Corresponding apparent mass densities are given in [Gong et al., 1964a], see Table 4.

Table 4: Bone compositions from dehydration-deorganification experiments of Gong et al. [1964a]

\begin{tabular}{|l|l|l|l|l|}
\hline \hline Tissue & $\rho^{e c}$ & $\rho_{\text {org }}^{*}$ & $\rho_{H_{2} O}^{*}$ & $\rho_{H A}^{*} A$ \\
\hline & $\begin{array}{l}{\left[\mathrm{g} / \mathrm{cm}^{3}\right]} \\
\text { given }\end{array}$ & $\begin{array}{l}{\left[\mathrm{g} / \mathrm{cm}^{3}\right]} \\
\text { given }\end{array}$ & $\begin{array}{l}{\left[\mathrm{g} / \mathrm{cm}^{3}\right]} \\
\text { given }\end{array}$ & $\begin{array}{c}{\left[\mathrm{g} / \mathrm{cm}^{3}\right]} \\
\text { Eq. (12) }\end{array}$ \\
\hline \hline Steer tibial shaft & 1.995 & 0.486 & 0.252 & 1.257 \\
Dog femoral shaft & 2.003 & 0.519 & 0.223 & 1.261 \\
Human femur and tibia & 1.991 & 0.476 & 0.237 & 1.278 \\
Monkey femur diaphysis & 2.035 & 0.487 & 0.239 & 1.309 \\
Steer atlas bone & 1.93 & 0.51 & 0.28 & 1.14 \\
Dog lumbar vertebral body & 1.91 & 0.51 & 0.29 & 1.11 \\
Human 12th thoracic, 1st and 2nd lumbar vertebral body & 1.92 & 0.50 & 0.27 & 1.16 \\
Monkey lumbar vertebrae & 1.88 & 0.51 & 0.27 & 1.09 \\
\hline
\end{tabular}

\subsection{Dehydration-ashing tests - Concentrations of hydroxyapatite, organics, and water}

According to the protocols of Burns [1929] and Hammett [1925], whole bones of rats with fresh weight $M_{\text {air }}$ are dried at 105-110 for 24 hours [Hammett, 1925; Chick et al., 1926] and at 96 for seven days [Hammett, 1925, 1924], respectively. Thereafter, the bones exhibit masses $M_{d r y}$. Through Eqs. (3) and (4), these masses give access to the water weight fractions $W F_{\mathrm{H}_{2} \mathrm{O}}$. Next, the dried bones are gently incinerated until all organic matter is burned off. Subsequent weighing results in the hydroxyapatite mass $M_{H A}$, giving access to mineral and organic weight fractions according to Eqs. (9), (8), and (6). These weight fractions, in turn, provide access to the mean mass density of the extracellular matrix of the investigated bones, through Eq. (16). Together with Eqs. (16), Eqs. (13)-(15) deliver the apparent mass densities of organics, water, and hydroxyapatite. Corresponding values are documented in Table 5. 
Table 5: Bone compositions from dehydration-ashing experiments of Hammett [1925] and Burns [1929]; THESE TISSUES WERE TAKEN FROM GROWING ORGANISMS, BEING IN THE STATES OF CHILDHOOD AND ADOLESCENCE

\begin{tabular}{|c|c|c|c|c|c|c|c|}
\hline Tissue & $W F_{H A}$ & $W F_{\text {org }}$ & $W F_{H_{2}} \mathrm{~d}$ & $\rho^{e c}$ & $\rho_{H A}^{*}$ & $\rho_{\text {org }}^{*}$ & $\rho_{\mathrm{H}_{2} \mathrm{O}}^{*}$ \\
\hline & $\begin{array}{l}- \\
\text { given } \\
\end{array}$ & $\begin{array}{l}- \\
\text { given } \\
\end{array}$ & $\begin{array}{l}- \\
\text { given } \\
\end{array}$ & $\begin{array}{l}{\left[\mathrm{g} / \mathrm{cm}^{3}\right]} \\
\text { Eq. (16) }\end{array}$ & $\begin{array}{l}{\left[\mathrm{g} / \mathrm{cm}^{3}\right]} \\
\text { Eq. }(10) \\
\end{array}$ & $\begin{array}{l}{\left[\mathrm{g} / \mathrm{cm}^{3}\right]} \\
\text { Eq. (7) }\end{array}$ & $\begin{array}{l}{\left[\mathrm{g} / \mathrm{cm}^{3}\right]} \\
\text { Eq. (5) }\end{array}$ \\
\hline Rabbit femur, fibula, tibia, humerus, radius and ulna ${ }^{a}$ & 0.31 & 0.23 & 0.45 & 1.381 & 0.428 & 0.324 & 0.628 \\
\hline Rabbit femur, fibula, tibia, humerus, radius and ulna $\mathrm{a}^{\mathrm{a}}$ & 0.21 & 0.20 & 0.59 & 1.250 & 0.266 & 0.246 & 0.737 \\
\hline Rat leg bones $^{\mathrm{a}}$ & 0.42 & 0.25 & 0.34 & 1.54 & 0.64 & 0.38 & 0.52 \\
\hline Rat leg bones ${ }^{\mathrm{a}}$ & 0.37 & 0.24 & 0.40 & 1.45 & 0.53 & 0.35 & 0.58 \\
\hline Rat leg bones ${ }^{\mathrm{a}}$ & 0.42 & 0.24 & 0.34 & 1.54 & 0.65 & 0.38 & 0.52 \\
\hline Rat leg bones ${ }^{\mathrm{a}}$ & 0.40 & 0.23 & 0.36 & 1.52 & 0.62 & 0.35 & 0.54 \\
\hline Rat leg bones ${ }^{\mathrm{a}}$ & 0.40 & 0.24 & 0.36 & 1.50 & 0.59 & 0.36 & 0.54 \\
\hline Humerus of rat ${ }^{\mathrm{b}}$ & 0.18 & 0.19 & 0.63 & 1.209 & 0.155 & 0.212 & 0.798 \\
\hline Humerus of rat ${ }^{b}$ & 0.18 & 0.19 & 0.63 & 1.213 & 0.169 & 0.225 & 0.784 \\
\hline Humerus of rat ${ }^{b}$ & 0.26 & 0.20 & 0.53 & 1.308 & 0.249 & 0.253 & 0.738 \\
\hline Humerus of rat ${ }^{b}$ & 0.32 & 0.21 & 0.48 & 1.372 & 0.379 & 0.290 & 0.668 \\
\hline Humerus of rat ${ }^{b}$ & 0.36 & 0.21 & 0.43 & 1.433 & 0.433 & 0.288 & 0.651 \\
\hline Humerus of rat ${ }^{b}$ & 0.42 & 0.22 & 0.36 & 1.524 & 0.509 & 0.311 & 0.610 \\
\hline Humerus of rat ${ }^{b}$ & 0.45 & 0.23 & 0.32 & 1.580 & 0.628 & 0.349 & 0.543 \\
\hline Humerus of rat ${ }^{\mathrm{b}}$ & 0.17 & 0.18 & 0.65 & 1.199 & 0.169 & 0.233 & 0.779 \\
\hline Humerus of rat ${ }^{b}$ & 0.18 & 0.19 & 0.63 & 1.209 & 0.178 & 0.231 & 0.777 \\
\hline Humerus of rat ${ }^{b}$ & 0.23 & 0.20 & 0.57 & 1.273 & 0.300 & 0.266 & 0.711 \\
\hline Humerus of rat ${ }^{b}$ & 0.31 & 0.21 & 0.48 & 1.372 & 0.387 & 0.285 & 0.669 \\
\hline Humerus of rat ${ }^{b}$ & 0.34 & 0.21 & 0.46 & 1.398 & 0.475 & 0.301 & 0.629 \\
\hline Humerus of rat ${ }^{b}$ & 0.38 & 0.21 & 0.41 & 1.459 & 0.601 & 0.333 & 0.564 \\
\hline Humerus of rat ${ }^{b}$ & 0.42 & 0.22 & 0.35 & 1.532 & 0.666 & 0.364 & 0.520 \\
\hline Femur of rat ${ }^{b}$ & 0.14 & 0.20 & 0.66 & 1.180 & 0.205 & 0.215 & 0.779 \\
\hline Femur of rat ${ }^{b}$ & 0.15 & 0.19 & 0.65 & 1.186 & 0.213 & 0.230 & 0.766 \\
\hline Femur of rat ${ }^{b}$ & 0.24 & 0.21 & 0.56 & 1.277 & 0.299 & 0.253 & 0.721 \\
\hline Femur of rat ${ }^{b}$ & 0.29 & 0.21 & 0.50 & 1.341 & 0.432 & 0.288 & 0.652 \\
\hline Femur of rat ${ }^{b}$ & 0.34 & 0.21 & 0.45 & 1.404 & 0.471 & 0.290 & 0.637 \\
\hline Femur of rat ${ }^{b}$ & 0.40 & 0.22 & 0.38 & 1.497 & 0.552 & 0.314 & 0.594 \\
\hline Femur of rat ${ }^{b}$ & 0.43 & 0.23 & 0.34 & 1.550 & 0.650 & 0.340 & 0.542 \\
\hline Femur of rat ${ }^{b}$ & 0.13 & 0.18 & 0.68 & 1.165 & 0.212 & 0.234 & 0.763 \\
\hline Femur of rat ${ }^{b}$ & 0.14 & 0.19 & 0.67 & 1.178 & 0.218 & 0.234 & 0.761 \\
\hline Femur of rat ${ }^{b}$ & 0.20 & 0.20 & 0.59 & 1.240 & 0.345 & 0.268 & 0.695 \\
\hline Femur of rat ${ }^{b}$ & 0.28 & 0.22 & 0.50 & 1.337 & 0.432 & 0.287 & 0.652 \\
\hline Femur of rat ${ }^{b}$ & 0.32 & 0.21 & 0.47 & 1.372 & 0.519 & 0.300 & 0.614 \\
\hline Femur of rat ${ }^{b}$ & 0.36 & 0.22 & 0.43 & 1.429 & 0.640 & 0.334 & 0.550 \\
\hline Femur of rat ${ }^{b}$ & 0,41 & 0.23 & 0.36 & 1.520 & 0.713 & 0.361 & 0.506 \\
\hline
\end{tabular}

b Hammett [1925]

According to the protocol of Biltz and Pellegrino [1969], bone samples with original weight of $M_{a i r}$, are dried at 105 until a constant mass, $M_{d r y}$, is attained. Thereafter, they are incinerated at 600 , for sixteen hours, being transformed into ash with weight $M_{a s h}$. Biltz and Pellegrino [1969] documented the volume fraction of water

$$
f_{H_{2} O}=\frac{\left(M_{a i r}-M_{d r y}\right)}{V^{e c}},
$$

as well as the weight fraction of ash in dried samples, 


$$
W F_{a s h}^{d r y}=\frac{M_{a s h}}{M_{d r y}} .
$$

Gong et al. [1964b] showed that, at 600 , not only organic matter, but also part of the hydroxyapatite becomes volatile during incineration. In quantitative terms, $6.6 \%$ of the ash weight is the weight of volatile inorganic. Hence, the weight fraction of hydroxyapatite in a dried bone sample reads as

$$
W F_{H A}^{d r y}=W F_{a s h}^{d r y} \times 1.066
$$

$W F_{H A}^{d r y}$ gives access to the apparent mass density of hydroxyapatite, via

$$
\rho_{H A}^{*}=W F_{H A}^{d r y} \times\left(\rho_{e x p}^{e c}-\rho_{H_{2} O}^{*}\right)
$$

with the apparent mass density of water following from

$$
\rho_{\mathrm{H}_{2} \mathrm{O}}^{*}=f_{\mathrm{H}_{2} \mathrm{O}} \times \rho_{\mathrm{H}_{2} \mathrm{O}}
$$

\begin{tabular}{|c|c|c|c|c|c|c|c|c|c|c|}
\hline Tissue & $\rho^{e c}$ & $f_{\mathrm{H}_{2} \mathrm{O}}$ & $W F_{a s h}^{d r y}$ & $\begin{array}{l}F_{H A}^{d r y} \\
\end{array}$ & $W F_{H A}^{e c}$ & $W F_{o r g}^{e c}$ & $W F_{\mathrm{H}_{2} \mathrm{O}}^{e c}$ & $\rho_{H A}^{*}$ & $\rho_{\text {org }}^{*}$ & $\rho_{\mathrm{H}_{2} \mathrm{O}}^{*}$ \\
\hline $\begin{array}{l}\text { Cortical bone of } \\
\text { femora and tibia: }\end{array}$ & $\begin{array}{l}{\left[\mathrm{g} / \mathrm{cm}^{3}\right]} \\
\text { given }\end{array}$ & given & $\begin{array}{l}- \\
\text { given }\end{array}$ & $\begin{array}{l}- \\
\text { Eq. (19) }\end{array}$ & $\begin{array}{l}- \\
\text { Eq. (14) }\end{array}$ & $\begin{array}{l}- \\
\text { Eq. (13) }\end{array}$ & $\begin{array}{l}- \\
\text { Eq. (15) }\end{array}$ & $\begin{array}{l}{\left[\mathrm{g} / \mathrm{cm}^{3}\right]} \\
\text { Eq. } \\
(20) \\
\end{array}$ & $\begin{array}{l}{\left[\mathrm{g} / \mathrm{cm}^{3}\right]} \\
\text { Eq. } \\
(12) \\
\end{array}$ & $\begin{array}{l}\left.\mathrm{g} / \mathrm{cm}^{3}\right] \\
\text { Eq. } \\
(21) \\
\end{array}$ \\
\hline Fish & 1.800 & 0.40 & 0.61 & 0.65 & 0.51 & 0.27 & 0.22 & 0.913 & 0.491 & 0.396 \\
\hline Turtle & 1.810 & 0.37 & 0.62 & 0.67 & 0.53 & 0.27 & 0.20 & 0.958 & 0.482 & 0.370 \\
\hline Frog & 1.930 & 0.35 & 0.66 & 0.70 & 0.57 & 0.25 & 0.18 & 1.103 & 0.475 & 0.352 \\
\hline Polar Bear & 1.920 & 0.33 & 0.66 & 0.70 & 0.58 & 0.25 & 0.17 & 1.119 & 0.471 & 0.330 \\
\hline Man & 1.940 & 0.15 & 0.67 & 0.71 & 0.66 & 0.26 & 0.08 & 1.275 & 0.510 & 0.155 \\
\hline Elephant & 2.000 & 0.20 & 0.69 & 0.73 & 0.66 & 0.24 & 0.10 & 1.316 & 0.484 & 0.200 \\
\hline Monkey & 2.090 & 0.23 & 0.69 & 0.73 & 0.65 & 0.24 & 0.11 & 1.364 & 0.496 & 0.230 \\
\hline Cat & 2.050 & 0.24 & 0.69 & 0.74 & 0.65 & 0.23 & 0.12 & 1.336 & 0.478 & 0.236 \\
\hline Horse & 2.020 & 0.25 & 0.69 & 0.74 & 0.65 & 0.23 & 0.12 & 1.309 & 0.461 & 0.250 \\
\hline Chicken & 2.040 & 0.24 & 0.70 & 0.74 & 0.65 & 0.23 & 0.12 & 1.332 & 0.463 & 0.245 \\
\hline Dog & 1.940 & 0.28 & 0.70 & 0.74 & 0.64 & 0.22 & 0.14 & 1.235 & 0.425 & 0.280 \\
\hline Goose & 2.040 & 0.23 & 0.71 & 0.75 & 0.67 & 0.22 & 0.11 & 1.365 & 0.445 & 0.230 \\
\hline Cow & 2.000 & 0.26 & 0.71 & 0.76 & 0.66 & 0.21 & 0.13 & 1.315 & 0.423 & 0.262 \\
\hline Guinea Pig & 2.100 & 0.25 & 0.71 & 0.76 & 0.67 & 0.21 & 0.12 & 1.404 & 0.446 & 0.250 \\
\hline Rabbit & 2.120 & 0.24 & 0.73 & 0.77 & 0.69 & 0.20 & 0.12 & 1.453 & 0.422 & 0.245 \\
\hline Rat & 2.240 & 0.20 & 0.73 & 0.78 & 0.71 & 0.20 & 0.09 & 1.597 & 0.441 & 0.202 \\
\hline
\end{tabular}

Finally, the apparent mass density of organic matter follows from Eq. (12). Corresponding values are documented in Table 6.

Table 6: Bone compositions from dehydration-ashing experiments of Biltz and Pellegrino [1969] 


\section{3. 'Universal' rules in bone fibrillogenesis and mineralization}

The concentrations (apparent mass densities) of organics, mineral, and water, in bone tissues from a great variety of species, organs, and ages [as determined through Eqs. (1) -(21)], strongly correlate mutually, and they also correlate with the bone tissue mass density, as seen in Figures 1-5, plotted on the basis of the data evaluated in Tables $1-6$.

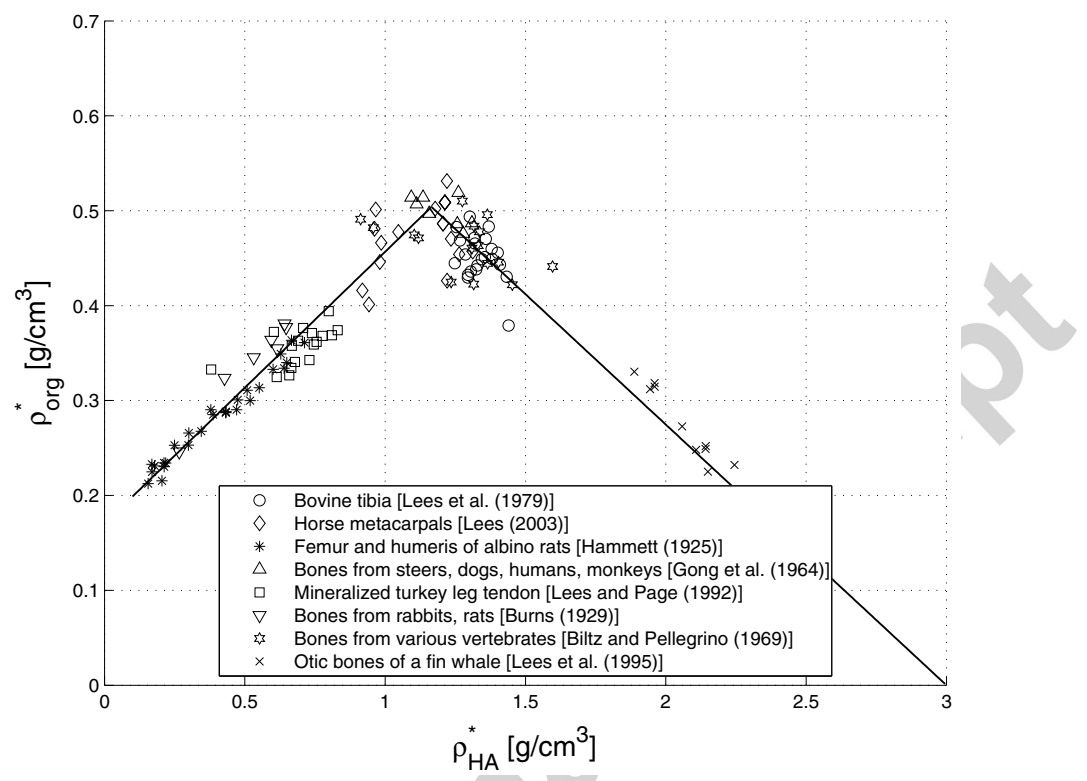

Figure 1: Relation between apparent mass densities of hydroxyapatite and organic matter in extracellular bone matrix, across different species, organs, and ages (see Tables 1 to 6 for numerical values and specification of bone types)

INTERESTINGLY, ALL THESE CORRELATIONS CAN BE REPRESENTED BY BILINEAR FUNCTIONS, WHEREBY THE INCREASING BRANCH DEPICTED IN FIGURE 1 RELATES TO TISSUES TAKEN FROM GROWING ORGANISMS (BEING IN THE STATES OF CHILDHOOD AND ADOLESCENCE), WHILE THE DECREASING BRANCH RELATES TO TISSUES TAKEN FROM ADULT ORGANISMS. As for the relationship between organics and mineral concentration, an increase of organic concentration with increasing mineral concentration (up to a critical value $\rho_{H A}^{*, \text { crit }}$ of about $1.20 \mathrm{~g} / \mathrm{m}^{3}$ ) is followed by a decrease of organic concentration with increasing mineral concentration (larger than about $1.20 \mathrm{~g} / \mathrm{m}^{3}$ ), see Figure 1. THE BILINEAR RELATIONSHIP CAN BE REPRESENTED BY TWO LINEAR BRANCHES, AN ASCENDING BRANCH FOR LOW MINERAL CONCENTRATIONS,

$$
\mathscr{F}_{\rho_{\text {org }}^{*}}\left(\rho_{H A}^{*}\right)=A \times \rho_{H A}^{*}+B \quad \text { FOR } 0<\rho_{H A}^{*} \leqslant \rho_{H A}^{*, c r i t}
$$

AND A DESCENDING BRANCH FOR HIGH MINERAL CONCENTRATIONS, WHICH IS A 


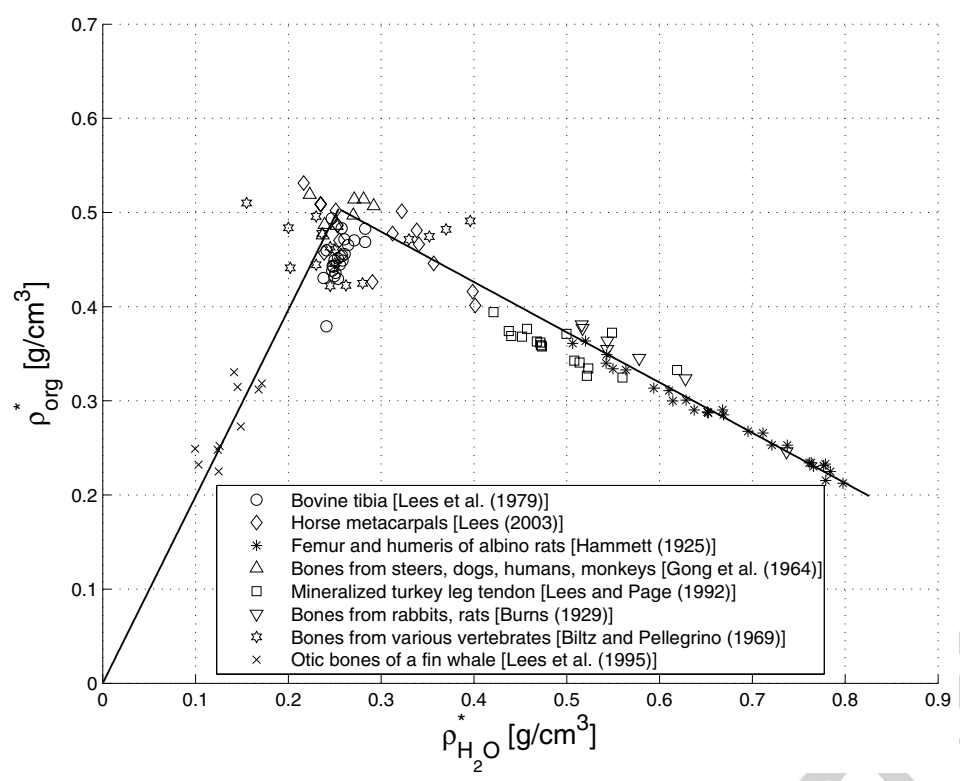

Figure 2: Relation between apparent mass densities of water and organic matter in extracellular bone matrix, across different species, organs, and ages (see Tables 1 to 6 for numerical values and specification of bone types)

FUNCTION OF THE PARAMETERS A AND B DEFINING THE ASCENDING BRANCH, AS WELL AS OF THE CRITICAL MINERAL CONCENTRATION $\rho_{H A}^{*, c r i t}$, AND OF THE REAL MASS DENSITY OF HYDROXYAPATITE, $\rho_{H A}=3$ G/ $\mathrm{CM}^{3}$ [GONG ET AL., 1964B; LEES, 1987; HELLMICH, 2005],

$$
\begin{aligned}
& \mathscr{F}_{\rho_{o r g}^{*}}\left(\rho_{H A}^{*}\right)=\left[A \times \rho_{H A}^{*, c r i t}+B\right] \times\left[1-\frac{\rho_{H A}^{*}-\rho_{H A}^{*, c r i t}}{\rho_{H A}-\rho_{H A}^{*, c r i t}}\right] \\
& \text { for } \rho_{H A}^{*, \text { crit }}<\rho_{H A}^{*}<\rho_{H A} \text {. }
\end{aligned}
$$

In Eq. (22) and (23), $A, B$, and $\rho_{H A}^{*, c r i t}$ are determined such that the sum of squares of the differences between the $N$ experimental values $\rho_{\text {org }, i}^{*}$ (corresponding to hydroxyapatite concentrations $\left.\rho_{H A, i}^{*}\right)$ and corresponding fitted values $\mathscr{F}_{\rho_{\text {org }}^{*}}\left(\rho_{H A, i}^{*}\right)$, according to Eqs. (22) and (23), is minimized,

$$
\mathfrak{F}\left(A, B, \rho_{H A}^{*, c r i t}\right)=\sum_{i=1}^{N}\left[\rho_{\text {org }, i}^{*}-\mathscr{F}_{\rho_{\text {org }}^{*}}\left(\rho_{H A, i}^{*}\right)\right]^{2} \rightarrow \text { min }
$$

This yields $A=0.29 \mathrm{~g} / \mathrm{cm}^{3}, B=0.17 \mathrm{~g} / \mathrm{cm}^{3}$, and $\rho_{H A}^{*, c r i t}=1.18 \mathrm{~g} / \mathrm{cm}^{3}$. The maximum apparent mass density of organic matter follows as $\mathscr{F}_{\rho_{\text {org }}^{*}}\left(\rho_{H A}^{*, \text { crit }}\right)=0.51 \mathrm{~g} / \mathrm{cm}^{3}$. 


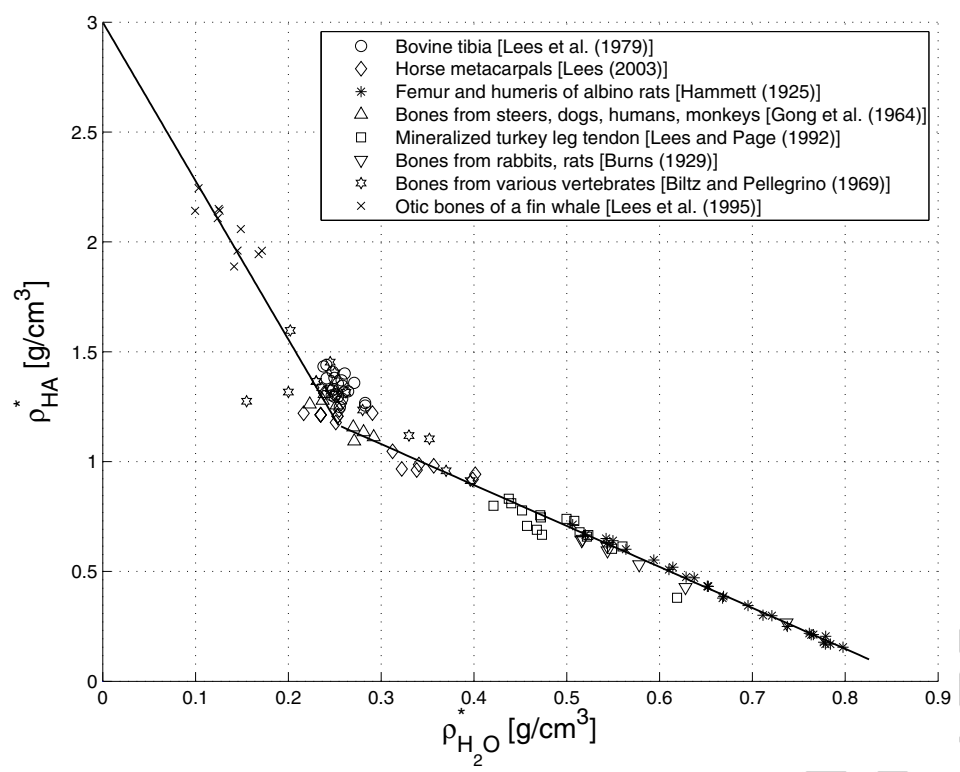

Figure 3: Relation between apparent mass densities of water and hydroxyapatite in extracellular bone matrix, across different species, organs, and ages (see Tables 1 to 6 for numerical values and specification of bone types)

The quality of the bilinear representation expressed through Eq. (22) and (23) is underlined by the coefficient of determination

$$
R^{2}=1-\left(\frac{S S_{e r r}}{S S_{t o t}}\right)
$$

with the total sum of squares

$$
S S_{t o t}=\sum_{i=1}^{N}\left(\rho_{o r g, i}^{*}-\frac{1}{N} \sum \rho_{o r g, i}^{*}\right)^{2}
$$

and the sum of squares of residuals

$$
S S_{\text {err }}=\sum_{i=1}^{N}\left[\rho_{\text {org }, i}^{*}-\mathscr{F}_{\rho_{\text {org }}^{*}}\left(\rho_{H A, i}^{*}\right)\right]^{2}
$$

amounting to $R^{2}=0.94$.

The apparent mass densities of hydroxyapatite and organics can be transformed into volume fractions (volume of hydroxyapatite or organics within a piece of extracellular 


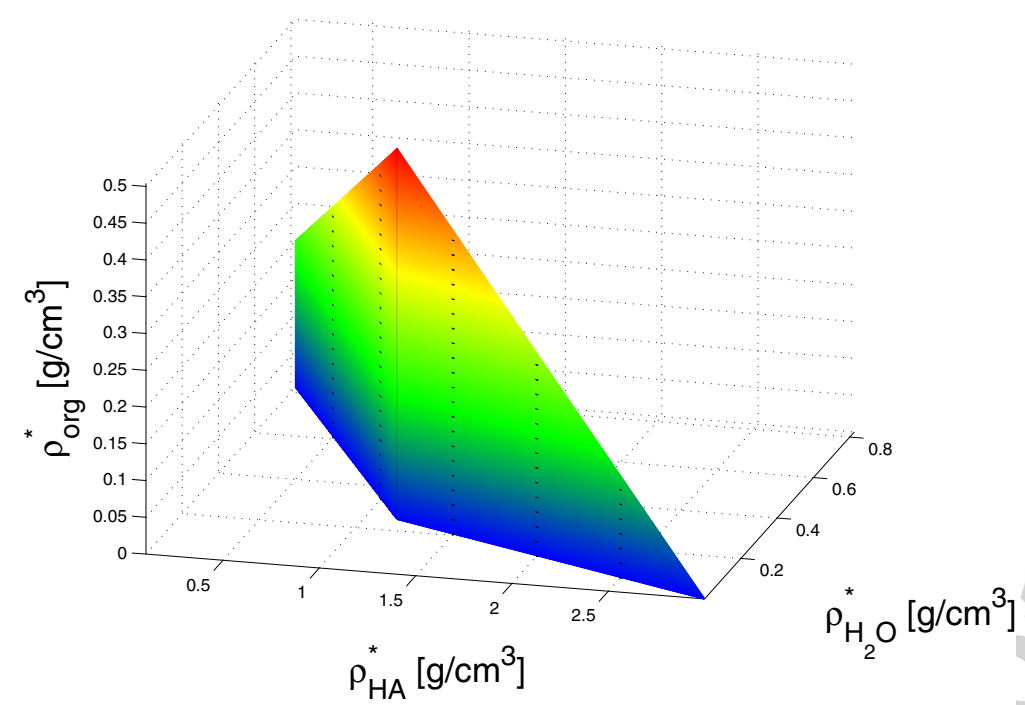

Figure 4: 3D representation of interrelations between hydroxapatite, organics, and water concentrations, in terms of apparent mass densities $\rho_{\mathrm{HA}}^{*}, \rho_{\mathrm{org}}^{*}$, and $\rho_{\mathrm{H}_{2} \mathrm{O}}^{*}$

bone matrix, over volume $V_{e c}$ of this piece), through

$$
\begin{aligned}
& f_{H A}=\frac{\rho_{H A}^{*}}{\rho_{H A}}, \\
& f_{\text {org }}=\frac{\rho_{o r g}^{*}}{\rho_{\text {org }}} .
\end{aligned}
$$

Since the remaining space in the aformentioned piece of extracellular bone tissue is occupied by water, the volume fraction of the latter follows as

$$
f_{H_{2} O}=1-f_{H A}-f_{\text {org }}
$$

which gives acess to the apparent mass density of water as

$$
\rho_{\mathrm{H}_{2} \mathrm{O}}^{*}=f_{\mathrm{H}_{2} \mathrm{O}} \times \rho_{\mathrm{H}_{2} \mathrm{O}} .
$$

When representing $\rho_{\text {org }}^{*}$ through function $\mathscr{F}_{\rho_{\text {org }}^{*}}$ [see Eqs. (22) and (23)], Eqs. (28) to (31) define bilinear relations involving the apparent mass density of water, $\rho_{\mathrm{H}_{2} \mathrm{O}}^{*}$, depicted in Figures 2 and 3. These relationships are characterized by coefficients of determination amounting to $R^{2}=0.75$ and $R^{2}=0.95$, respectively. FOR THE SAKE OF A COMPACT ILLUSTRATION, THE BILINEAR RELATIONS OF FIGURES 1, 2, AND 

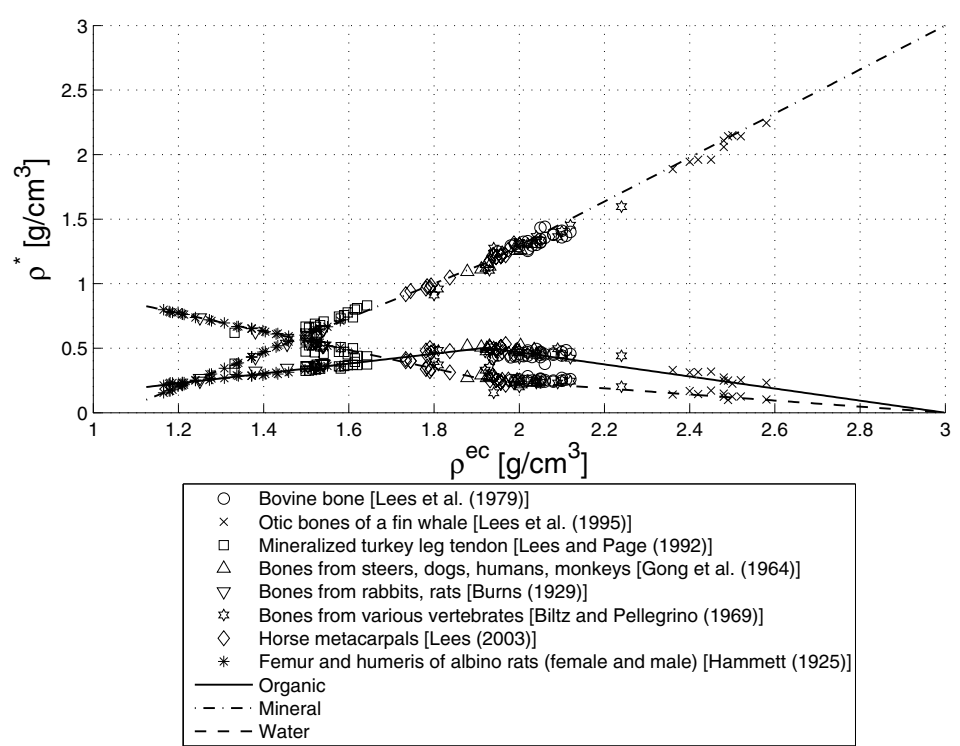

Figure 5: Apparent mass densities of water, hydroxyapatite, and organic matter, versus overall mass density $\rho^{e c}$ of extracellular bone matrix, across different species, organs, and ages (see Tables 1 to 6 as in Figures 1 to 3

3, Are simultaneously depicted in a 3D plot, see Figure 4. Alternatively, the apparent mass densities can be given as functions of the extracellular bone tissue mass density

$$
\rho^{e c}=f_{H_{2} O} \times \rho_{H_{2} O}+f_{o r g} \times \rho_{\text {org }}+f_{H A} \times \rho_{H A},
$$

see Figure 5. Corresponding coefficients of determination for water, organics, and hydroxyapatite amount to $R^{2}=0.97, R^{2}=0.90$, and $R^{2}=0.99$, respectively. Alternatively, with the help of Eqs. (28), (29), and (30), the extracellular mass density $\rho^{e c}$ can be related to volume fractions of hydroxyapatite, organics, and water (see Figure 6).

\section{4. 'Universal' relations between extracellular mass density and tissue elasticity}

Recently, hierarchical material models for bone [Hellmich et al., 2004; Fritsch and Hellmich, 2007; Fritsch et al., 2009a], developed within the framework of continuum micromechanics [Zaoui, 2002] and validated through a multitude of biochemical, biophysical, and biomechanical experiments [Bonar et al., 1985; Lees, 1987; Ashman et al., 1984; McCarthy et al., 1990], have opened the way to translate the chemical composition of extracellular bone material (i.e. the volume fractions of organics, water, and hydroxyapatite, as seen in Figure 6) into the tissue's anisotropic elasticity. In 


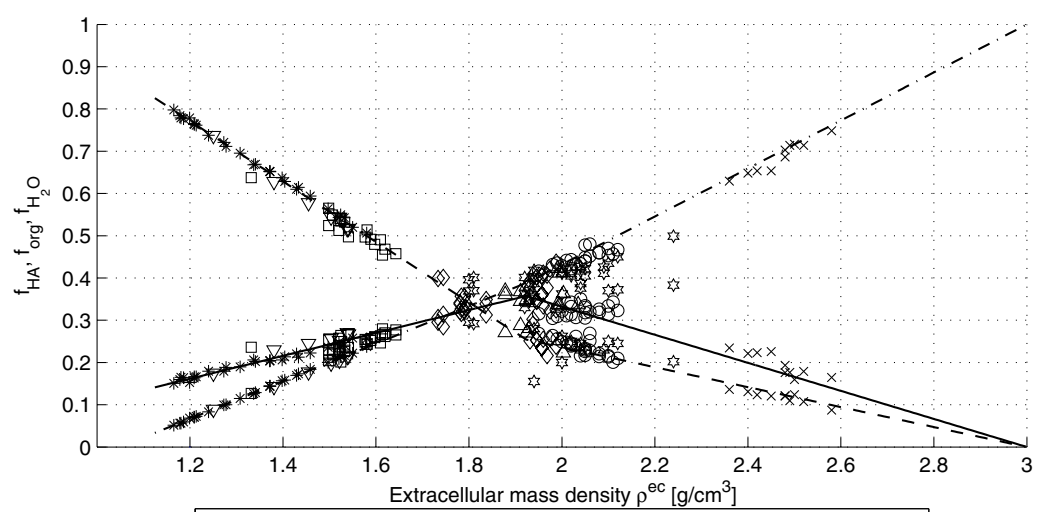

\begin{tabular}{|cl|}
\hline$\circ$ & Bovine bone [Lees et al. (1979)] \\
$\times$ & Otic bones of a fin whale [Lees et al. (1995)] \\
$\square$ & Mineralized turkey leg tendon [Lees and Page (1992)] \\
$\triangle$ & Bones from steers, dogs, humans, monkeys [Gong et al. (1964)] \\
$\nabla$ & Bones from rabbits, rats [Burns (1929)] \\
$\vdots$ & Bones from various vertebrates [Biltz and Pellegrino (1969)] \\
$\diamond$ & Horse metacarpals [Lees (2003)] \\
$*$ & Femur and humeris of albino rats (female and male) [Hammett (1925)] \\
\hline- & Organic \\
-- & Mineral \\
- & - Water \\
\hline
\end{tabular}

Figure 6: Volume fractions of hydroxyapatite, organs, and water in extracellular bone matrix, as function of the mass density of the latter

the following, we will combine the aforementioned micromechanics models with the relations depicted in Figure 6, as to provide 'universal' relations between extracellular mass density and tissue elasticity (such relations are of interest for biomaterial design or simulation-based biomedical engineering, as discussed later, see Section 5). In more detail, we employ the four-step elastic homogenization scheme reported in [Fritsch and Hellmich, 2007; Fritsch et al., 2009a], see Figure 7, based on the tissue-independent 'universal' elastic properties of the elementary building blocks of extracellular bone material, namely hydroxyapatite, collagen, and water with some insignificant amount of non-collagenous organics. These properties, given in [Hellmich et al., 2004; Fritsch and Hellmich, 2007], are accessible through ultrasonic techniques [Katz and Ukraincik, 1971; Cusack and Miller, 1979] or ab initio simultations [Ching et al., 2009]. The aformentioned multilevel homogenization scheme relates the stiffness of material phases (i.e. quasi-homogeneous subdomains) within a representative volume element (RVE) [e.g. molecular collagen within RVE of wet collagen in Figure 7(a), or mineralized collagen fibril within RVE of extracellular bone matrix in Figure 7(d)], to the stiffness of the RVE itself, as a function of the phase stiffnesses and of the phase volume fractions in all RVEs. On a mathematical level, this is achieved by setting the phase strains equal to those in ellipsoidal inclusions in infinitely extending matrices of stiffness $\mathbb{C}^{0}$ subjected to remote strains, and by combining respective semi-analytical relationships [Eshelby, 1957; Laws, 1977] with stress and strain average rules [Hashin, 1983; Zaoui, 2002]. For all four RVEs depicted in Figure 7, the phase elasticities are related to the overall RVE-specific ("homogenized") elasticity through the standard ex- 
pression of matrix-inclusion-problem-based continuum micromechanics [Benveniste, 1987; Zaoui, 2002]

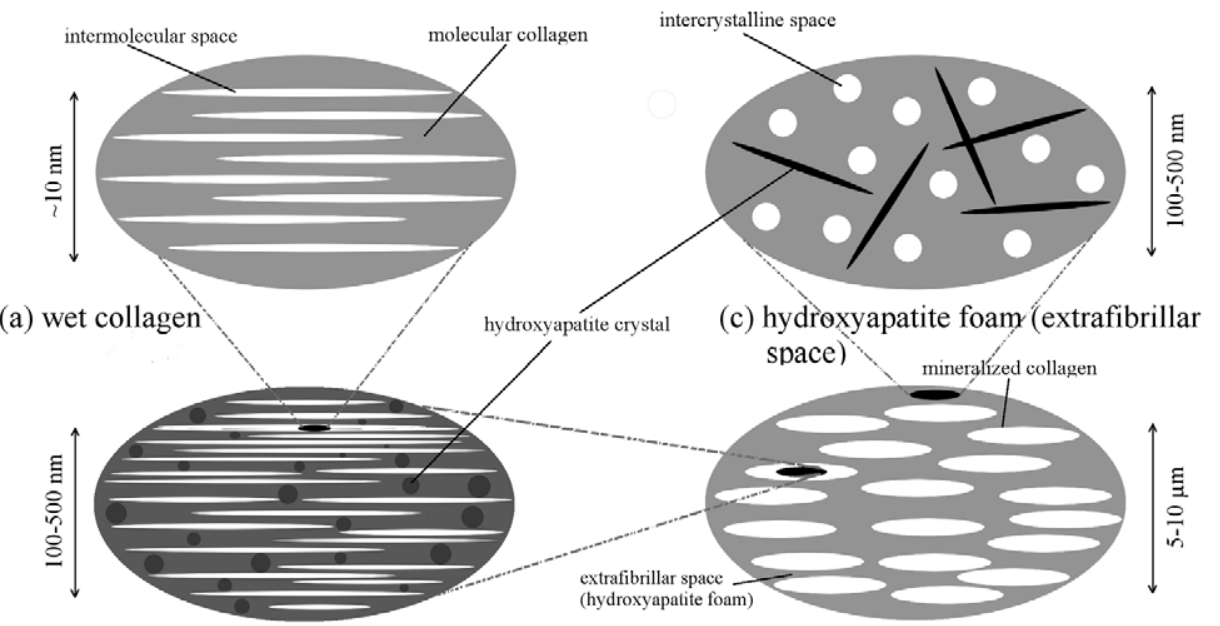

(b) collagen-hydroxyapatite network (fibril)

(d) extracellular bone matrix

Figure 7: Four-step homogenization scheme after Fritsch and Hellmich [2007], Fritsch et al. [2009a]

$$
\begin{aligned}
\mathbb{C}^{h o m}= & \sum_{r} f_{r} \mathbb{C}_{r}:\left[\mathbb{I}+\mathbb{P}_{r}^{0}:\left(\mathbb{C}_{r}-\mathbb{C}^{0}\right)\right]^{-1} \\
& :\left\{\sum_{s} f_{s}\left[\mathbb{I}+\mathbb{P}_{s}^{0}:\left(\mathbb{C}_{s}-\mathbb{C}^{0}\right)\right]^{-1}\right\}^{-1},
\end{aligned}
$$

where $f_{r}$ and $\mathbb{C}_{r}$ are the volume fraction and the elastic stiffness of phase $r, \mathbb{I}$ is the fourth-order unity tensor, $\mathbb{P}_{r}^{0}$ the fourth-order Hill tensor accounting for the characteristic shape of phase $r$, and $\mathbb{C}^{0}$ is the matrix stiffness. Choice of this stiffness describes the interactions between the phases: for $\mathbb{C}^{0}$ coinciding with one of the phase stiffnesses [Mori-Tanaka scheme, [Mori and Tanaka, 1973; Wakashima and Tsukamoto, 1991]], a composite material is represented [contiguous matrix with inclusions - see Figure 7(d)]; for $\mathbb{C}^{0}=\mathbb{C}^{\text {hom }}$ (self-consistent scheme Hershey [1954]; Hill [1963]), a dispersed arrangement of the phases is considered [typical for polycrystals - see Figure $7(a, b, c)])$. In case of the extrafibrillar space [Figure 7(c)], with infinitely many crystal phases oriented in all directions, the sums in Eq. (33) need to be replaced by integrals over the unit sphere, as detailled in Fritsch et al. [2009a,b].

The volume fractions of the four RVEs of Figure 7 need to be derived from $f_{\text {org }}$, $f_{\mathrm{HA}}$, and $f_{\mathrm{H}_{2} \mathrm{O}}$ : first, the volume fraction of collagen is determined from the organic volume fraction, through the fact [Urist et al., 1983; Lees, 1987; Buckwalter et al., 1995 ] that $90 \%$ of the organic matter in extracellular bone matrix is collagen, 


$$
f_{\text {col }}=0.9 \times f_{\text {org }} .
$$

The volume fraction of the fibrils and the extrafibrillar space [see Figure 7 (b,c,d)], $f_{f i b}$ and $f_{e f}$ can be quantified on the basis of the generalized packing model of Lees et al. [1984b]; Lees [1987], through

$$
f_{f i b}=f_{c o l} \times \frac{v_{f i b}}{v_{c o l}}, \quad v_{f i b}=b \times d_{s} 5 D,
$$

where $f_{c o l}$ is determined according to Eq. (34). In Eq. (35), $v_{c o l}=335.6 \mathrm{~nm}^{3}$ is the volume of a single collagen molecule [Lees, 1987]; $v_{f i b}$ is the volume of one rhomboidal fibrillar unit with length $5 D$, width $b$, and height $d_{s} ; b=1.47 \mathrm{~nm}$ is an average (rigid) collagen crosslink length valid for all mineralized tissues [Lees et al., $1984 \mathrm{~b}$ ], $D \approx 64 \mathrm{~nm}$ is the axial macroperiod of staggered assemblies of type I collagen [Hodge and Petruska, 1963], and $d_{s}$ is the tissue-specific neutron diffraction spacing between collagen molecules, which depends on the mineralization and the hydration state of the tissue [Lees et al., 1984a; Bonar et al., 1985; Lees et al., 1994b]. For wet tissues, $d_{s}$ can be given in a dimensionless form [Hellmich and Ulm, 2003], as a function of $\rho^{e c}$ only

$$
d_{s}=\text { const } \times m_{0}^{\frac{1}{3}} \rho^{e c^{-\frac{1}{3}}},
$$

for $\left(\right.$ const $\left.\times m_{0}^{\frac{1}{3}}\right)=1.57 \times 10^{-10} \mathrm{~kg}^{\frac{1}{3}}$.

The volume fractions for scales below the ultrastructure can be derived directly from $f_{f i b}$ and $f_{c o l}$, on the basis of the finding of Hellmich and Ulm [2001, 2003] that the average hydroxyapatite concentration in the extra-collagenous space of the extracellular wet mineralized tissues is the same inside and outside the fibrils. Accordingly, the relative amount of hydroxyapatite in the extrafibrillar space reads as [Hellmich and Ulm, 2001, 2003]

$$
\phi_{H A, e f}=\frac{1-f_{f i b}}{1-f_{c o l}} .
$$

With this value at hand, the mineral volume fractions in the fibrillar and the extrafibrillar space follow as [see Figure 7 (b,c,d)]

$$
\begin{aligned}
& \breve{f}_{H A}=\frac{f_{H A}\left(1-\phi_{H A, e f}\right)}{f_{f i b}}, \\
& \check{f}_{H A}=\frac{\phi_{H A, e f} f_{H A}}{f_{e f}} .
\end{aligned}
$$


Within the fibril, comprising the phases hydroxyapatite and wet collagen [see Figure 7 (b)], the volume fraction of the latter reads as

$$
\breve{f}_{\text {wetcol }}=1-\breve{f}_{H A} .
$$

Finally, the volume fraction of (molecular) collagen [see Figure 7(a)] at the wet collagen level can be calculated from $f_{c o l}$, through

$$
\stackrel{\circ}{\text { col }}=\frac{f_{\text {col }}}{\breve{f}_{\text {wetcol }}} .
$$

Eqs. (33) and (36), together with Eqs. (34)-(41), deliver the extracellular elasticity tensor components $C_{i j k l}^{e c}$ as functions of the extracellular mass density $\rho^{e c}$ [see Figures 9 (a) and 9(b)]. These elasticity tensor components give access to the transverse and longitudinal Young's moduli via

$$
\begin{aligned}
& E_{1}^{e c}=\frac{1}{D_{1111}^{e c}}, \\
& E_{3}^{e c}=\frac{1}{D_{3333}^{e c}},
\end{aligned}
$$

with compliance tensor $\mathbb{D}^{e c}$ being the inverse of $\mathbb{C}^{e c}$, see Figures 10(a) and 10(b) for mass density-moduli relations; the transverse and longitudinal Poisson's ratios, as well as the in-plane shear modulus of the bone material read as

$$
\begin{aligned}
& \nu_{12}^{e c}=-D_{1122}^{e c} \times E_{1}^{e c}, \\
& \nu_{13}^{e c}=-D_{1133}^{e c} \times E_{3}^{e c}, \\
& G_{12}^{e c}=\frac{E_{1}^{e c}}{2\left(1+\nu_{12}^{e c}\right)}=C_{1212}^{e c},
\end{aligned}
$$

see Figures 11(a), 11(b), 10(a) and 10(b) for the dependencies of these quantities on the extracellular mass density.

These mass density-elasticity relations can be independently checked through ultrasonic experiments at a frequency of $10 \mathrm{MHz}$ [Lees et al., 1979b, 1983, 1995], revealing the elastic properties of extracellular bone matrix as [Carcione, 2001; Fritsch and Hellmich, 2007] 


$$
\begin{aligned}
& C_{1111}^{e c}=\rho^{e c} v_{1}^{2}, \\
& C_{3333}^{e c}=\rho^{e c} v_{3}^{2},
\end{aligned}
$$

with $v_{1}$ and $v_{3}$ being the velocities of the longitudinal acoustic waves travelling in the transverse and axial directions, respectively, see Tables 7 and 8 for experimental values. The mean $\bar{e}$ and the standard deviation $e_{s}$ of the relative errors between $N$ stiffness predictions and experiments,

$$
\begin{aligned}
& \bar{e}=\frac{1}{N} \sum e_{i}=\frac{1}{N} \sum \frac{C_{i j k l}^{p r e d}-C_{i j k l}^{e x p}}{C_{i j k l}^{e x p}}, \\
& e_{s}=\left[\frac{1}{N-1} \sum\left(e_{i}-\bar{e}\right)^{2}\right]^{1 / 2},
\end{aligned}
$$

are as low as $+5.47 \pm 10.17 \%$ [mean value \pm standard deviation] for the transverse normal stiffness $C_{1111}^{e c}$, and $+4.82 \pm 8.81 \%$ [mean value \pm standard deviation] for the longitudinal normal stiffness $C_{3333}^{e c}$, see Figure 8 for a comparison of model predictions and experiments. This is, to the authors' knowledge, the highest predictive precision ever attained in the field of bone microelasticity. The mass density-elasticity relationship of Figure 9 can be suitably approximated through higher order polynomials with mean relative errors below $0.25 \%$ (with respect to the micromechanical estimates). In a dimensionless form based on the normal elastic stiffness and the mass density of hydroxyapatite, $C_{1111}^{H A}=137 \mathrm{GPa}$ [Katz and Ukraincik, 1971] and $\rho_{H A}=3 \mathrm{~g} / \mathrm{cm}^{3}$ [Lees, 1987], such polynomials read as

$$
\begin{aligned}
C_{1111}^{e c}\left(\rho^{e c}\right) / C_{111}^{H A} \simeq & +4.6826 \times\left(\rho^{e c} / \rho_{H A}\right)^{3}-6.0171 \times\left(\rho^{e c} / \rho_{H A}\right)^{2} \\
& +2.8081 \times\left(\rho^{e c} / \rho_{H A}\right)-0.4470,
\end{aligned}
$$

with a relative approximation error of $-0.17 \pm 1.64 \%$ [mean value \pm standard deviation];

$$
\begin{aligned}
C_{3333}^{e c}\left(\rho^{e c}\right) / C_{1111}^{H A} \simeq & -6.8447 \times\left(\rho^{e c} / \rho_{H A}\right)^{4}+17.6300 \times\left(\rho^{e c} / \rho_{H A}\right)^{3} \\
& -13.5048 \times\left(\rho^{e c} / \rho_{H A}\right)^{2}+4.2118 \times\left(\rho^{e c} / \rho_{H A}\right) \\
& -0.4573,
\end{aligned}
$$

with a relative approximation error of $+0.2 \pm 5.5 \%$; 


$$
\begin{aligned}
C_{1122}^{e c}\left(\rho^{e c}\right) / C_{1111}^{H A} \simeq & -11.0152 \times\left(\rho^{e c} / \rho_{H A}\right)^{5}+29.7474 \times\left(\rho^{e c} / \rho_{H A}\right)^{4} \\
& -28.7144 \times\left(\rho^{e c} / \rho_{H A}\right)^{3}+12.5870 \times\left(\rho^{e c} / \rho_{H A}\right)^{2} \quad(53) \\
& -2.3375 \times\left(\rho^{e c} / \rho_{H A}\right)+0.1188
\end{aligned}
$$

with a relative approximation error of $-0.02 \pm 0.69 \%$;

$$
\begin{aligned}
C_{1133}^{e c}\left(\rho^{e c}\right) / C_{1111}^{H A} \simeq & -5.0088 \times\left(\rho^{e c} / \rho_{H A}\right)^{4}+13.7237 \times\left(\rho^{e c} / \rho_{H A}\right)^{3} \\
& -12.4876 \times\left(\rho^{e c} / \rho_{H A}\right)^{2}+4.8307 \times\left(\rho^{e c} / \rho_{H A}\right) \\
& -0.6745,
\end{aligned}
$$

with a relative approximation error of $-0.05 \pm 2.42 \%$; and finally

$$
\begin{aligned}
C_{2323}^{e c}\left(\rho^{e c}\right) / C_{1111}^{H A} \simeq & +4.1245 \times\left(\rho^{e c} / \rho_{H A}\right)^{5}-14.9352 \times\left(\rho^{e c} / \rho_{H A}\right)^{4} \\
& +21.9578 \times\left(\rho^{e c} / \rho_{H A}\right)^{3}-15.1486 \times\left(\rho^{e c} / \rho_{H A}\right)^{2}(55) \\
& +4.9459 \times\left(\rho^{e c} / \rho_{H A}\right)-0.6169
\end{aligned}
$$

with a relative approximation error of $-0.18 \pm 5.02 \%$. 
$\times \quad C_{1111}:$ Bovine bone - [Lees et al. (1979)]

$\triangleright C_{1111}$ : Whale bones - [Lees et al. (1995)]

$\diamond C_{1111}$ : Bones from various vertebrates - [Lees et al. (1983)]

- $\mathrm{C}_{3333}$ : Bovine bone - [Lees et al. (1979)]

- $\mathrm{C}_{3333}$ : Bones from various vertebrates - [Lees et al. (1983)]

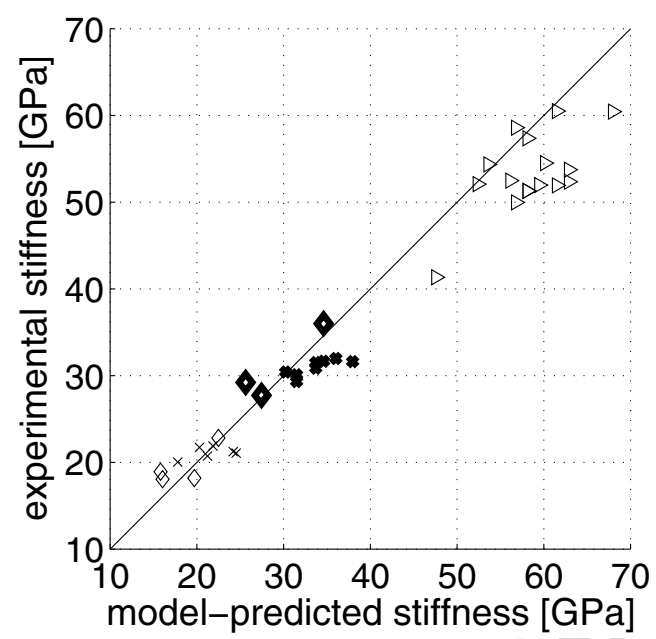

Figure 8: Extracellular tissue elasticity: Comparison between model-predicted stiffness values and data from ultrasonic experiments at $10 \mathrm{MHz}$ frequency

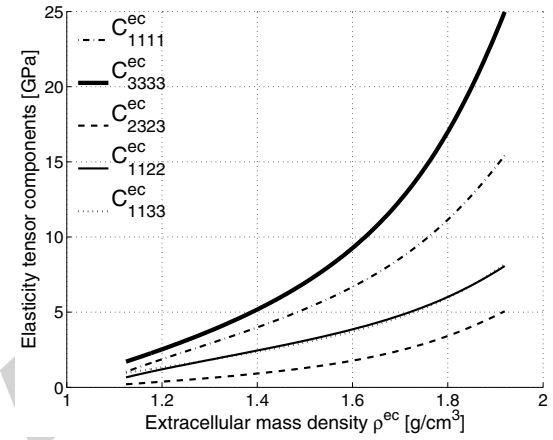

(a) Low mass density

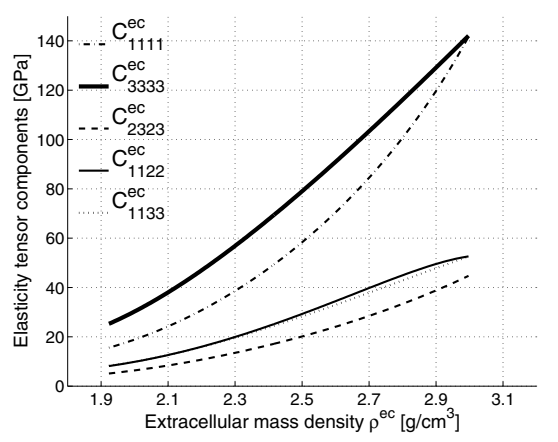

(b) High mass density

Figure 9: Elasticity tensor components of extracellular bone matrix, as functions of the mass density of the latter ( $3 \ldots$ axial direction; $1,2 \ldots$ transverse direction) 
Table 7: Experimental ultrasound data in transverse direction

\begin{tabular}{|c|c|c|c|c|}
\hline Tissue & $\rho^{e c}$ & $\nu_{1}$ & $C_{1111, e x p}^{e c}$ & $C_{1111, p r e d}^{e c}$ \\
\hline & $\begin{array}{l}{\left[\mathrm{g} / \mathrm{cm}^{3}\right]} \\
\text { given } \\
\end{array}$ & $\begin{array}{l}{[\mathrm{m} / \mathrm{s}]} \\
\text { given }\end{array}$ & $\begin{array}{l}\text { [GPa] } \\
\text { Eq. (47) }\end{array}$ & $\begin{array}{l}\text { [GPa] } \\
\text { Eqs. (33)-(41) }\end{array}$ \\
\hline Bovine tibia $^{\mathrm{a}}$ & 2.10 & 3.18 & 21.2 & 24.2 \\
\hline Bovine tibia ${ }^{\mathrm{a}}$ & 1.98 & 3.18 & 20.0 & 17.8 \\
\hline Bovine tibia $^{a}$ & 2.05 & 3.18 & 20.7 & 21.2 \\
\hline Bovine tibia ${ }^{a}$ & 2.11 & 3.16 & 21.1 & 24.5 \\
\hline Bovine tibia $^{a}$ & 2.03 & 3.27 & 21.7 & 20.3 \\
\hline Bovine tibia $^{\mathrm{a}}$ & 2.06 & 3.26 & 21.9 & 21.9 \\
\hline Bovine tibia ${ }^{b}$ & 2.07 & 3.32 & 22.8 & 22.5 \\
\hline Dugong rib ${ }^{b}$ & 2.02 & 3.00 & 18.2 & 19.7 \\
\hline Elephant radius ${ }^{b}$ & 1.94 & 3.05 & 18.0 & 16.0 \\
\hline Human femur ${ }^{b}$ & 1.93 & 3.13 & 18.9 & 15.8 \\
\hline Whale malleus ${ }^{c}$ & 2.49 & 4.85 & 58.6 & 56.8 \\
\hline Whale malleus ${ }^{c}$ & 2.53 & 4.89 & 60.5 & 61.5 \\
\hline Whale malleus ${ }^{c}$ & 2.51 & 4.55 & 52.0 & 59.5 \\
\hline Whale malleus $c$ & 2.45 & 4.61 & 52.1 & 52.4 \\
\hline Whale incus ${ }^{c}$ & 2.50 & 4.79 & 57.4 & 58.1 \\
\hline Whale incus ${ }^{c}$ & 2.46 & 4.70 & 54.3 & 53.6 \\
\hline Whale stapes ${ }^{c}$ & 2.40 & 4.15 & 41.3 & 47.6 \\
\hline Whale stapes ${ }^{c}$ & 2.48 & 4.60 & 52.5 & 56.1 \\
\hline Whale periotic ${ }^{c}$ & 2.50 & 4.53 & 51.3 & 58.1 \\
\hline Whale periotic ${ }^{c}$ & 2.52 & 4.65 & 54.5 & 60.1 \\
\hline Whale periotic ${ }^{\mathrm{c}}$ & 2.58 & 4.84 & 60.4 & 68.0 \\
\hline Whale periotic ${ }^{c}$ & 2.54 & 4.60 & 53.7 & 62.9 \\
\hline Whale periotic ${ }^{c}$ & 2.50 & 4.53 & 51.3 & 58.1 \\
\hline Whale t. bulla ${ }^{\mathrm{c}}$ & 2.53 & 4.53 & 51.9 & 61.5 \\
\hline Whale t. bulla ${ }^{\mathrm{c}}$ & 2.54 & 4.54 & 52.4 & 62.9 \\
\hline Whale t. bulla ${ }^{\mathrm{c}}$ & 2.49 & 4.48 & 50.0 & 56.8 \\
\hline
\end{tabular}

Table 8: Experimental ultrasound data in axial direction

\begin{tabular}{|l|l|l|l|l|}
\hline \hline Tissue & $\rho^{e c}$ & $\nu_{3}$ & $C_{3333, e x p}^{e c}$ & $C_{3333, p r e d}^{e c}$ \\
\hline & $\begin{array}{l}{\left[\mathrm{g} / \mathrm{cm}^{3}\right]} \\
\text { given }\end{array}$ & $\begin{array}{l}{[\mathrm{m} / \mathrm{s}]} \\
\text { given }\end{array}$ & $\begin{array}{l}{[\mathrm{GPa}]} \\
\text { Eq. (48) }\end{array}$ & [GPa] \\
Eqs. (33)-(41)
\end{tabular}




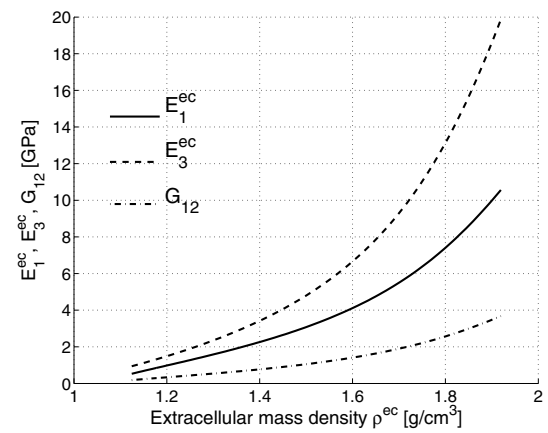

(a) Low mass density

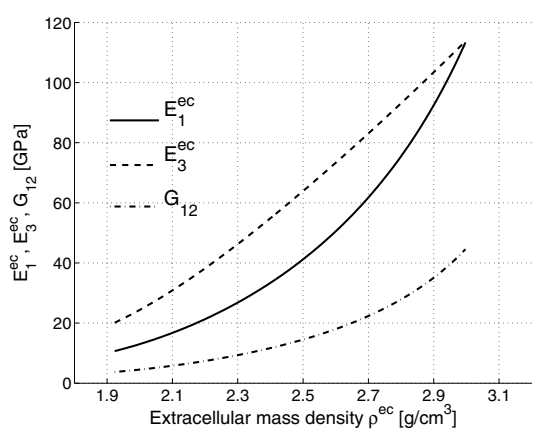

(b) High mass density

Figure 10: Young's and shear moduli of extracellular bone matrix, as functions of the mass density of the latter ( $3 \ldots$ axial direction; $1,2 \ldots$ transverse direction)

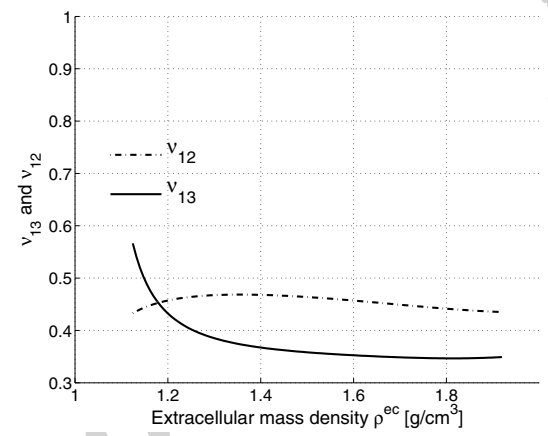

(a) Low mass density

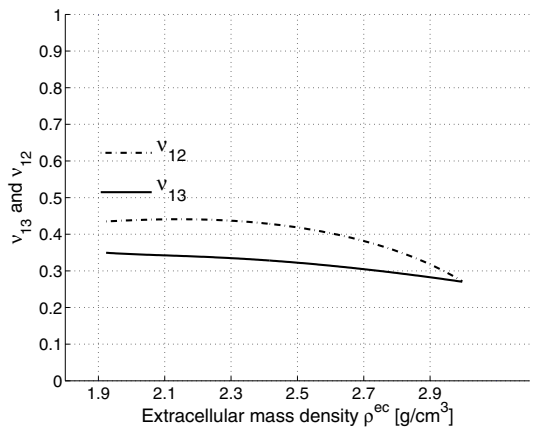

(b) High mass density

Figure 11: Poisson's ratios of extracellular bone matrix, as functions of the mass density of the latter (3 ....axial direction; $1,2 \ldots$ transverse direction) 


\section{Discussion}

This contribution revealed inter-relation between the mineral, organics, and water concentrations in extracellular bone materials, inherent to tissues across different species, organs, and ages, see Figures 1-6. In this context, it is particularly noteworthy that the positive progression along the $\rho_{H A}^{*}$-axis in Figure 1, as well as along the $\rho^{e c}$-axis in Figure 5, RELATES FIRST TO GROWING AND ADOLESCENCE (UP TO THE MAXIMUM ORGANIC CONCENTRATION), AND THEN TO FURTHER AGING OF THE BONE TISSUES DURING ADULTHOOD OF THE RESPECTIVE ANIMALS OR HUMANS. In fact, the data of Hammett [1925] for rat femur and humeris allow for computation of an organic apposition rate, as $\left(d \rho_{\text {org }}^{*} / d t\right)=0.0317 \mathrm{~g} / \mathrm{cm}^{3}$ per month, see Figure 12(a), which is even constant throughout the time of animal growth, as is the growth rate in rat tail tendon, see Figure 12(b) for data of Parry and Craig [1978].

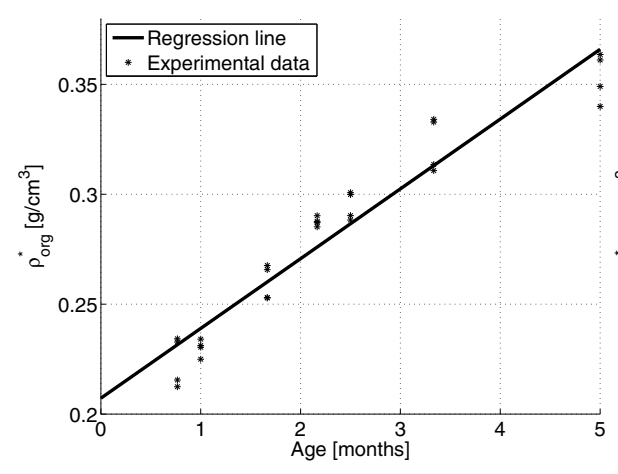

(a) Humeris and femur of an albino rat [Hammett, 1925]

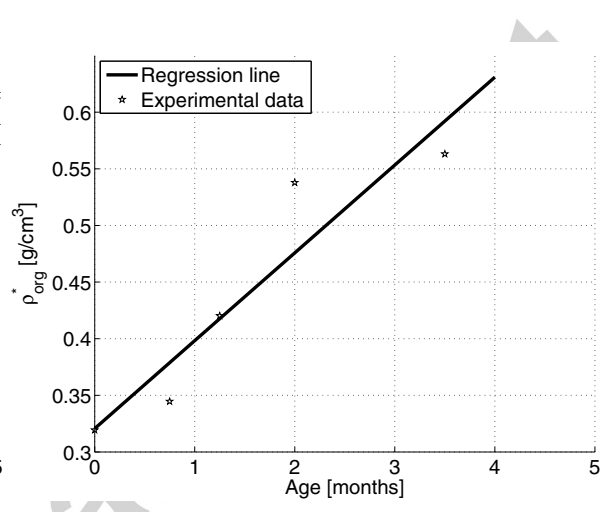

(b) Rat-Tail Tendon [Parry and Craig, 1978]

Figure 12: Age-related organic apparent mass density

Thus, while organic matter is accumulated during animal or human growth (see e.g. rising branch in Figure 1), it may be continuously reduced once adulthood is reached (see e.g. declining branch in Figure 1). This reduction, however, may be considerably delayed, i.e. occuring only at very high age in specific organs, since it is known from imaging techniques, such as computerized quantitative contact microradiography [Boivin and Meunier, 2002], quantitative backscattered electron imaging [Roschger et al., 2003], Raman microscopy [Akkus et al., 2003], and Synchroton Micro Computer Tomography [Bossy et al., 2004], that the chemical composition of adult bone matrix (when averaged over a millimeter-sized domain) remains, for a long time, constant throughout specific organs, and in particular with age [Hellmich et al., 2008]. During this time span, measured mechanical properties of the extracellular bone matrix, such as indentation modulus and hardness, appear also as time-invariant [Weaver, 1966; Hoffler et al., 2000; Wolfram et al., 2010; Rho et al., 2002; Burket et al., 2011], while such mechanical properties increase during animal (or human) growth [Feng and Jasiuk, 2011; Weaver, 1966]. It is interesting to discuss the mineral-organics concentration relation of Figure 1 from the viewpoint of cell biology: During growth, the 
mineral-to-organic mass apposition ratio in extracellular bone tissue is a constant

$$
\frac{d \rho_{H A}^{*}}{d \rho_{\text {org }}^{*}}=\frac{1}{A}=3.5 \quad \text { for } 0<\rho_{H A}^{*} \leqslant \rho_{H A}^{*, c r i t},
$$

"universally" valid throughout different tissues of different growing species at different ages, see Figure 1. This constant reflects the working mode of osteoblasts (CUBOIDAL OR POLYGONAL BONE CELLS WITH SEVERAL TENS OF MICROMETERS CHARACTERISTIC LENGTH [AARON, 1976; PARFITT, 1983; ROHOLl ET AL., 1994; JiLKA ET AL., 1998; ZHU ET AL., 2001; Noble, 2008; BONEWALD AND JOHNSON, 2008]. Pre-osteoblasts [Parfitt, 1983; Engler et AL., 2006; Lemaire ET AL., 2004] DEPOSIT NEW OSTEOID, IN THE FORM OF SEAMS OF SOME 8 TO 10 MICRONS THICKNESS, MADE OF PROTEOGLYCAN GEL REINFORCED BY FAIRLY RANDOMLY ORIENTED COLLAGEN FIBRILS [ENGLER ET AL., 2006; ZAJAC AND Discher, 2008; Buxboim AND Discher, 2010], SEe Fig. 13(A).

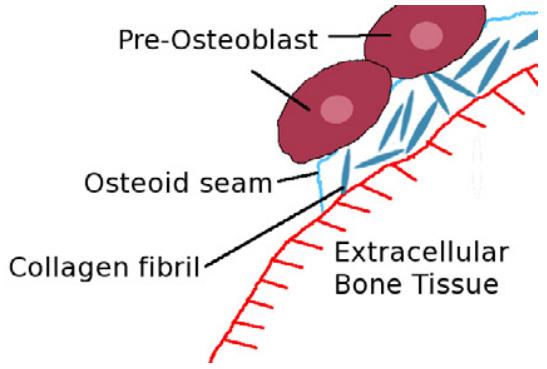

(a) Pre-Osteoblasts lay down an osteoid seam, reinforced by randomly oriented collagen fibrils

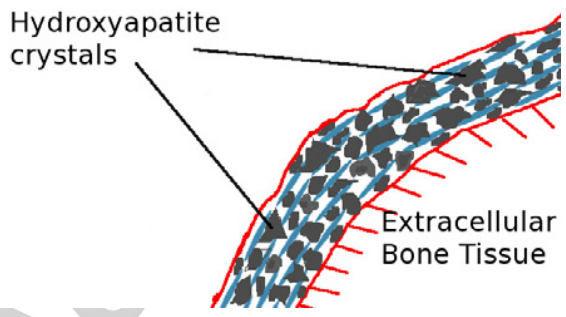

(c) Secondary mineralization: crystals grow without control of local biological cells

Figure 13: Working mode of pre-osteoblasts and osteoblasts: Primary and secondary mineralization of an unmineralized osteoid

THEREAFTER, OSTEOBLASTS ORDER THE COLLAGEN FIBRILS THROUGH STRETCHING [ENGLER ET AL., 2006], AND MEDIATE, THROUGH BUDDING OF 
MATRIX VESICLES FROM CELL PROCESSES, THE PRECIPITATION OF HYDROXYAPATITE, SEE FIgURE 13(B). THIS RESUlTS IN THE SO-CALlED PRIMARY MINERALIZATION [PARFITT, 1983], WITH A CHARACTERISTIC TIME OF HOURS TO DAYS [WERGEDAL AND BAYLINK, 1974]. FROM A CHEMICAL VIEWPOINT, SPECIALLY SYNTHESIZED MATRIX MOLECULES, SUCH AS BONE SIALOPROTEIN, OSTEOPONTIN, OR OSTEOCALCIN (WIESMANN ET AL, 2005), INDUCE MINERAL FORMATION, AND SUCH NON-COLLAGENOUS ORGANIC MOLECULES TYPICALLY MAKE UP 10\% OF THE OVERALL ORGANIC VOLUME FRACTION [URIST ET AL., 1983; LEES, 1987; BUCKWALTER ET AL., 1995], REGARDLESS OF THE MAGNITUDE OF THE LATTER. ACCORDINGLY, ONE WOULD EXPECT THE MORE MINERAL PRECIPITATION, THE MORE NON-COLLAGENEOUS ORGANICS PRESENT, THE AMOUNT OF THE LATTER BEING PROPORTIONAL TO THAT OF THE OVERALL ORGANIC MATTER - AND THIS IS PERFECTLY CONSISTENT WITH THE TISSUE- AND SPECIES-INDEPENDENT, “UNIVERSAL” MINERAL-PER-ORGANICS APPOSITION RATIO OF 3.5 IN EQ. (56), SUGGESTING, THEREFORE, PRIMARY MINERALIZATION AS THE DOMINANT MINERALIZATION MECHANISM IN GROWING ORGANISMS. IN THIS LINE, CALCIUM AND PHOSPHATE CONCENTRATIONS ARE NOT ONLY TIGHTLY REGULATED IN HUMANS AND OTHER VERTEBRATES, BUT THEY ARE PROBABLE TO FOLLOW EVEN SPECIES-INDEPENDENT CONCENTRATION RULES.

THESE ARGUMENTS, REFERRING TO PROPERTIES DEFINED PER VOLUME OF EXTRACELLULAR BONE TISSUE, ARE DECOUPLED FROM THE QUESTION ON HOW MUCH NEW OSTEOID IS LAID DOWN. TURNING TO THE LATTER ASPECT, THE MOST ABUNDANT BIOLOGICAL BONE CELLS, WHICH ARE THE OSTEOCYTES [BAYLINK AND Wergedal, 1971; AAron, 1976; PARFitT, 1983; Teitelbaum, 2000; NoBLE, 2008; BELL ET AL., 2008; BONUCCI, 2009] RESIDING IN THE LACUNAR POROSITY OF EXTRAVASCULAR BONE MATRIX, COME INTO PLAY. ORIGINITATING FROM OSTEOBLASTS WHICH WERE BURIED IN THE COURSE OF ONGOING OSTEOID FORMATION AND MINERALIZATION, OSTEOCYTES MAINTAIN A WIDELY SPREAD NETWORK, THROUGH CHANNELS CALLED CANALICULI, AMONG THEMSELVES AND WITH THE OSTEOBLASTS LOCATED AT THE BONE TISSUE SURFACE. THIS NETWORK IS THOUGHT TO EFFECTIVELY TRANSFER MECHANICAL STIMULI RELATED TO TISSUE DEFORMATION, TO THE OSTEOBLASTS [COWIN, 2007; BONEWALD AND JOHNSON, 2008], SO AS TO TRIGGER THEIR BONE FORMATION ACTIVITY, AS DESCRIBED BEFORE.

IN ADDITION TO MECHANOSENSING, OSTEOCYTES MAY INHIBIT MINERALIZATION AROUND THEIR LACUNAE [BONUCCI, 2009], AND THEREFORE SET AN UPPER LIMIT TO THE ASYMPTOTIC MINERAL CONCENTRATION WHICH MAY BE ATTAINED DURING THE PROCESS CALLED SECONDARY MINERALIZATION. THIS PROCESS EXHIBITS A CHARACTERISTIC TIME OF WEEKS TO MONTHS [BALA ET AL., 2010], SEE FIGURE 13(C), AND BEFORE REACHING ITS ASYMPTOTE, SECONDARY MINERALIZATION IS NOT CONTROLLED BY THE LOCAL BIOLOGICAL CELLS, BUT BY THE DIFFUSION AND COMPOSITION PROPERTIES OF THE FLUIDS SATURATING THE EXTRACEllular BONE tissue [PARFitT, 1983]. HoweVer, AT Higher AGES, THE AFOREMENTIONED INHIBITIVE ACTIVITY OF OSTEOCYTES STEADILIY DECREASES, SO THAT, IN THE END, EVEN THE LACUNAE THEMSELVES MAY BE FILLED WITH MINERAL, AS EVIDENCED BY FROST [1960], JOWSEY [1960], AND 
BELL ET AL. [2008]. As A CONSEQUENCE, THE ORGANIC-TO-MINERAL CONCENTRATION RATIO DECREASES, AND EQ. (56) IS NOT VALID ANY MORE. AT THE SAME TIME, OSTEOBLASTIC ACTIVITY ALSO DECREASES AT MORE ADVANCED AGES [AARON, 1976], LEADING TO A REDUCTION OF THE (ABSOLUTE) ORGANIC CONCENTRATION IN EXTRACELLULAR BONE MATRIX. THIS COMBINED EFFECT OF BOTH OSTEOBLASTIC AND OSTEOCYTIC ACTIVITY REDUCTION IS EXPRESSED BY A (NEGATIVE) MINERAL GROWTH-TO-ORGANIC-REMOVAL RATIO,

$$
\frac{d \rho_{H A}^{*}}{d \rho_{\text {org }}^{*}}=-3.6 \quad \text { FOR } \rho_{H A}^{*, c r i t}<\rho_{H A}^{*} \leqslant \rho_{H A},
$$

SEE Figure 1, WHich REVEALS SECONDARY MINERALIZATION AS THE DOMINANT MINERALIZATION MECHANISM IN ADULT, AGING ORGANISMS. WE ALSO REMARK THAT FIGURES 1-6 REFER TO PHYSIOLOGICALLY NORMAL CONDITIONS, WHILE DRUG TREATMENTS [LEES ET AL., 1994A] MAY LEAD TO CONSIDERABLE DEVIATIONS FROM THESE RULES FOR FIBRILLOGENESIS AND MINERALIZATION. WE OBSERVE A DECREASED ORGANIC-TO-MINERAL RATIO IN FIGURE 14, WHEREBY THE EXTENT OF THE DECREASE DEPENDS ON THE ADMINISTERED DRUGS: THE MAXIMUM DECREASE IS OBSERVED FOR FLUORIDE, FOLLOWED BY THOSE RELATED TO CORTISOL AND BAPN ( $\beta$-AMINOPROPIONITRILE).

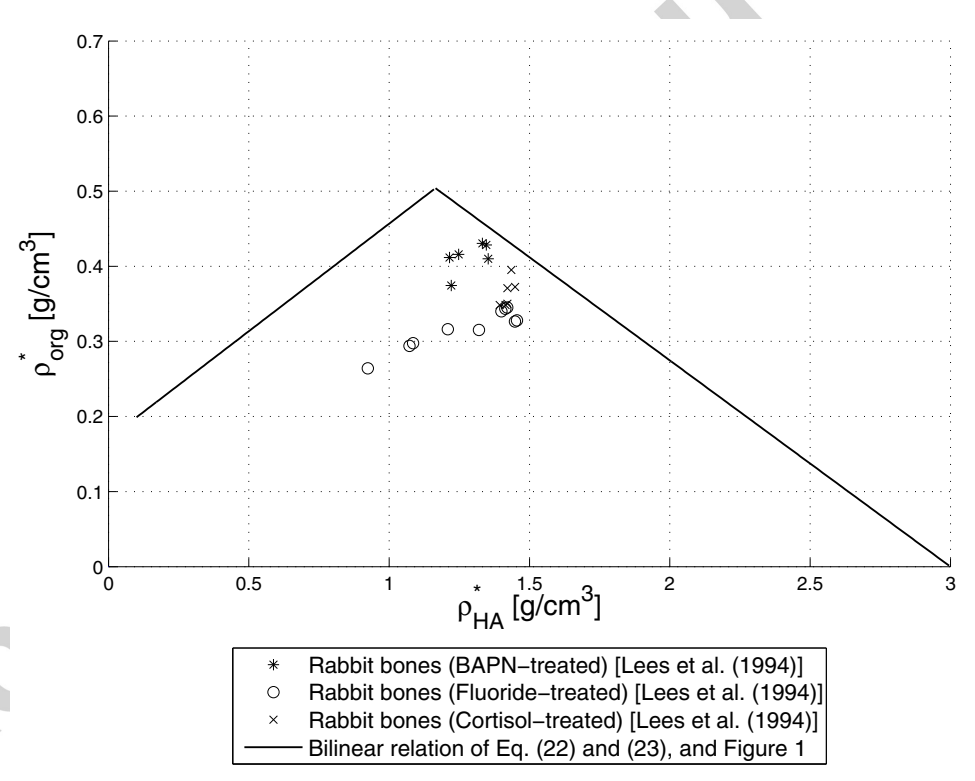

Figure 14: Mineral-to-organic concentration relations in extracellular bone tissue of drug-treated rabbits of Lees et al. [1994a] 


\section{Outlook}

WE ENVISION THE DISCOVERED CONCENTRATION RELATIONS OF FIGURES 16 AND 9-11, TO BE USEFUL IN VARIOUS CONTEXTS, SUCH AS (I) DEFINING THE SUCCESSION OF LIFETIME-VARIANT EXTRACELLULAR TISSUE COMPOSITIONS RELATED TO BONE REMODELING EQUILIBRIUM UNDER NORMAL PHYSIOLOGICAL CONDITIONS, WHICH COULD CONSTITUTE AN INTERESTING INPUT TO CORRESPONDING MATHEMATICAL BIOLOGY MODELS [LEMAIRE ET AL., 2004; PIVONKA ET AL., 2010]; AND (II) ENHANCED EXPLOITATION OF COMPUTER TOMOGRAPHIC (CT) DATA [KOMLEV ET AL., 2010] FOR BIOMECHANICAL ENGINEERING. Regarding the latter item, the volume fractions of the tissue's constituents can be related to the $\mathrm{X}$-ray attenuation coefficients quantifiable in a micro Computer Tomograph [Jackson and Hawkes, 1981; Hellmich et al., 2008; Scheiner et al., 2009]

$$
\mu^{e c}=f_{\mathrm{H}_{2} \mathrm{O}} \times \mu_{\mathrm{H}_{2} \mathrm{O}}+f_{\mathrm{HA}} \times \mu_{\mathrm{HA}}+f_{\text {org }} \times \mu_{\text {org }},
$$

with $\mu_{H_{2} O}=5.33 \mathrm{~cm}^{-1}, \mu_{\text {org }}=5.71 \mathrm{~cm}^{-1}, \mu_{H A}=142 \mathrm{~cm}^{-1}$, as the attenuation coefficients of water, organics, and hydroxyapatite, at a photon energy of $10 \mathrm{keV}$, which is typically used in bone micro CT imaging. The values for $\mu_{\mathrm{H}_{2} \mathrm{O}}, \mu_{\mathrm{HA}}$, and $\mu_{\text {org }}$ are accessible from the NIST database ${ }^{1}$. Combination of Eq. (58) with the miromechanics model Eqs. (33)-(41) yields attenuation coefficient-elasticity relations, see Fig. 15. They open interesting possibilities for CT-based, micromechanics-supported biomaterial design [Bertrand and Hellmich, 2009]: Voxel-specific gray values guide the way to individuum- and location-specific elastic properties, which an implant is required to exhibit, as not to disturb the physiological force field, necessary for undisturbed biophysical functionality of the organism.

\footnotetext{
${ }^{1}$ NIST XCOM database, US National Institute of Standards and Technology, http://physics.nist.gov/PhysRefData/Xcom/html/xcoml.html, 2010
} 


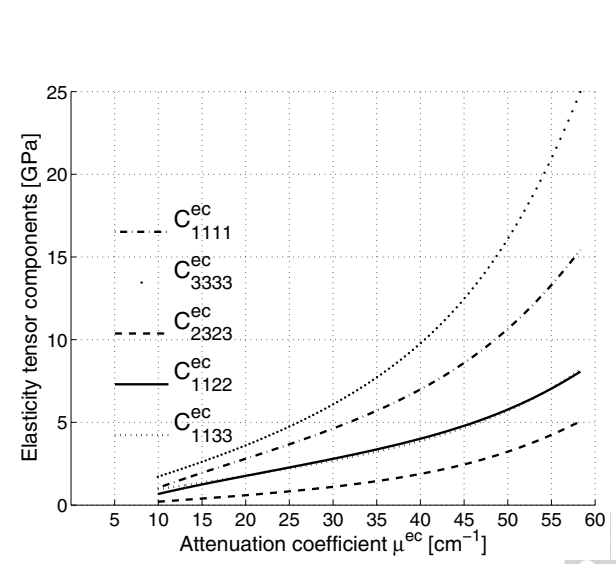

(a) Growing bone material

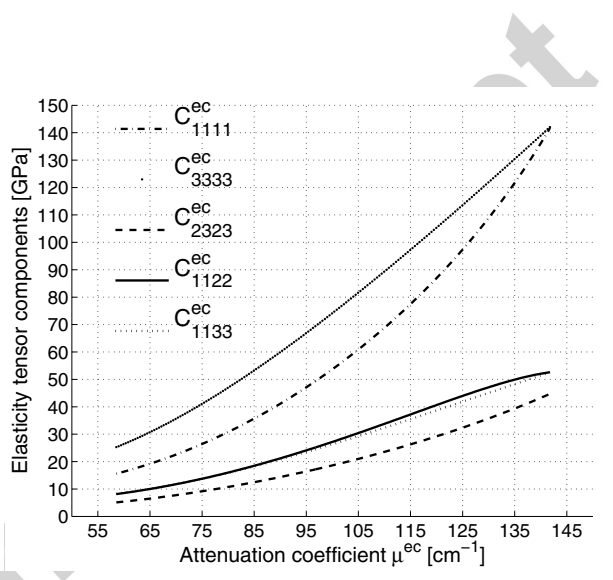

(b) Aging bone material

Figure 15: Elasticity tensor components as functions of attenuation coefficient $\mu^{e c}$ at $10 \mathrm{keV}$ photon energy (3 ... axial direction; $1,2 \ldots$ transverse direction) 


\section{Acknowledgments}

This work was partially supported by the European Commission under the Theme FP7-2008-SME-1 of the $7^{\text {th }}$ Framework Program, project no. 232 164, BIO-CTEXPLOIT. The authors gratefully acknowledge the support of Andreas Fritsch. 


\section{References}

Aaron, J. (1976). Histology and micro-anatomy of bone. In Nordin, B., editor, Calcium, Phosphate and Magnesium Metabolism: Clinical Physiology and Diagnostic Procedures, pages 298-356. Churchill Livingstone, New York.

Akkus, O., Polyakova-Akkus, A., Adar, F., and Schaffler, M. (2003). Aging of microstructural compartments in human compact bone. Journal of Bone and Mineral Research, 18(6):1012 - 1019.

Ashman, R., Cowin, S., van Buskirk, W., and Rice, J. (1984). A continuous wave technique for the measurement of the elastic properties of cortical bone. Journal of Biomechanics, 17(5):349 - 361.

Bala, Y., Farlay, D., Delmas, P., Meunier, P., and Boivin, G. (2010). Time sequence of secondary mineralization and microhardness in cortical and cancellous bone from ewes. Bone, 46:1204-1212.

Baylink, D. and Wergedal, J. (1971). Bone formation and resorption by osteocytes, pages 257-289. Academic Press, New York, USA.

Bell, L., Kayser, M., and Jones, C. (2008). The mineralized osteocyte: a living fossil. American Journal of Physical Anthropolgy, 137:449-456.

Benveniste, Y. (1987). A new approach to the application of Mori-Tanaka's theory in composite materials. Mechanics of Materials, 6(2):147-157.

Bertrand, E. and Hellmich, C. (2009). Multiscale elasticity of tissue engineering scaffolds with tissue-engineered bone: A continuum micromechanics approach. Journal of Engineering Mechanics (ASCE), 135:395-412.

Biltz, R. and Pellegrino, E. (1969). The chemical anatomy of bone. Journal of Bone and Joint Surgery, 51-A(3):456-466.

Boivin, G. and Meunier, P. (2002). The degree of mineralization of bone tissue measured by computerized quantitative contact microradiography. Calcified Tissue International, 70:503-511.

Bonar, L., Lees, S., and Mook, H. (1985). Neutron diffraction studies of collagen in fully mineralized bone. Journal of Molecular Biology, 181:265 - 270.

Bonewald, L. and Johnson, M. (2008). Osteocytes, mechanosensing and wnt signaling. Bone, 42(4):606 - 615 .

Bonucci, E. (2009). The osteocyte: the underestimated conductor of the bone orchestra. Rendiconti Lincei: Scienze Fisiche e Naturali, 20:237-254.

Bossy, E., Talmant, M., Peyrin, F., Akrout, L., Cloetens, P., and Laugier, P. (2004). In in vitro study of the ultrasonic axial transmission technique at the radius: $1 \mathrm{MHz}$ velocity measurements are sensitive to both mineralization and introcortical porosity. Journal of Bone and Mineral Research, 19(9):1548 - 1556. 
Broz, J. J., Simske, S. J., and Greenberg, A. R. (1995). Material and compositional properties of selectively demineralized cortical bone. Journal of Biomechanics, 28(11): 1357 - 1368 .

Buckwalter, J., Glimcher, M., Cooper, R., and Recker, R. (1995). Bone biology, Part I: Structure, blood supply, cells, matrix, and mineralization. The Journal of Bone and Joint Surgery, 77-A(8):1256 - 1275.

Burket, J., Gourion-Arsiquaud, S., Havill, L., Baker, S., Boskey, A., and van der Meulen M.C.H. (2011). Microstructure and nanomechanical properties in osteons relate to tissue and animal age. Journal of Biomechanics, 44:277 - 284.

Burns, C. M. (1929). XCIV. The effect of the continued ingestion of mineral acid on growth of body and bone and on the composition of bone and of the soft tissues. Biochemical Journal, 23(5):860 - 867.

Buxboim, A.and Ivanovska, I. and Discher, D. (2010). Matrix elasticity, cytoskeletal forces and physics of the nucleus: how deeply do cells 'feel' outside and in? Journal of Cell Science, 123(3):297-308.

Cancedda, R., Giannoni, P., and Mastrogiacomo, M. (2007). A tissue engineering approach to bone repair in large animal models and in clinical practice. Biomaterials, 28:4240 - 4250 .

Carcione, J. (2001). Wave fields in real media: Wave propagation in anisotropic, anelastic and porous media. In Helbig, K. and Treitel, S., editors, Handbook of Geophysical Exploration Seismic Exploration, volume 31. Elsevier, Amsterdam, The Netherlands.

Chick, H., Korenchevsky, V., and Roscoe, M. (1926). LXXXIII. The difference in chemical composition of the skeletons of young rats fed (1) on diets deprived of fatsoluble vitamins and (2) on a low phosphorus rachitic diet, compared with those of normally nourished animals of same age. Biochemical Journal, 20(3):621 - 631.

Ching, W., Rulis, P., and Misra, A. (2009). Ab initio elastic properties and tensile strength of crystalline hydroxyapatite. Acta Biomaterialia, 5:3067 - 3075.

Cowin, S. (2007). The significance of bone microstructure in mechanotransduction. Journal of Biomechanics, 40(Supplement 1):S105 - S109.

Cusack, S. and Miller, A. (1979). Determination of the elastic constants of collagen by Brillouin light scattering. Journal of Molecular Biology, 135:39 - 51.

Engler, A., Sen, S., Sweeney, H., and Discher, D. (2006). Matrix elasticity directs stem cell lineage specification. Cell, 126(4):677-689.

Eshelby, J. (1957). The determination of the elastic field of an ellipsoidal inclusion, and related problems. Proceedings of the Royal Society of London. Series A, Mathematical and Physical Sciences, 241(1226):376 - 396. 
Feng, L. and Jasiuk, I. (2011). Multi-scale characterization of swine femoral cortical bone. Journal of Biomechanics, 44:313 - 320.

Ficai, A., Andronescu, E., Voicu, G., Manzu, D., and Ficai, M. (2009). Layer by layer deposition of hydroxyapatite onto the collagen matrix. Materials Science and Engineering: C, 29(7):2217 - 2220.

Filvaroff, E. and Derynck, R. (1998). Bone remodelling: a signalling system for osteoclast regulation. Current Biology, 8:R679 - R682.

Fritsch, A., Dormieux, L., Hellmich, C., and Sanahuja, J. (2009a). Mechanical behaviour of hydroxyapatite biomaterials: An experimentally validated micromechanical model for elasticity and strength. Journal of Biomedical Materials Research, 88A:149-161.

Fritsch, A. and Hellmich, C. (2007). 'Universal' microstructural patterns in cortical and trabecular, extracellular and extravacular bone materials: Micromechanicsbased prediction of anisotropic elasticity. Journal of Theoretical Biology, 244:597 620 .

Fritsch, A., Hellmich, C., and Dormieux, L. (2009b). Ductile sliding between mineral crystals followed by rupture of collagen crosslinks: experimentally supported micromechanical explanation of bone strength. Journal of Theoretical Biology, 260:230-252.

Frost, H. (1960). Micropetrosis. Journal of Bone and Joint Surgery, 42:144 - 150.

Gajjeraman, S., Narayanan, K., Hao, J., Qin, C., and George, A. (2007). Matrix macromolecules in hard tissues control the nucleation and hierarchical assembly of hydroxyapatite. The Journal of Biologial Chemistry, 282(2):1193 - 1204.

Gong, J., Arnold, J., and Cohn, S, H. (1964a). Composition of trabecular and cortical bone. The Anatomical Record, 149:325 - 332.

Gong, J., Arnold, J., and Cohn, S, H. (1964b). The density of organic and volatile and non-volatile inorganic components of the bone. The Anatomical Record, 149:319324.

Gould, S. and Lewontin, R. (1979). The spandrels of san marco and the panglossian paradigm: A critique of the adaptationist programme. Proceedings of the Royal Society of London, Series B, 205(1161):581-598.

Green, D. (2008). Tissue bionics: examples in biomimetic tissue engineering. Biomedical Materials, 3:1-11.

Hammett, F. S. (1924). Studies of the thyroid apparatus: XIV. the growth of the humerus and femur of male and female albino rats thyro-parathyroidectomized and parathyroidectomized when 100 days of age. The Journal of Experimental Zoology, 39(3):465 - 504 . 
Hammett, F. S. (1925). A biochemical study of bone growth. I. Changes in the ash, organic matter and water during growth (mus norvegicus albinus). The Journal of Biological Chemistry, 64:409-428.

Hashin, Z. (1983). Analysis of composite materials - a survey. Journal of Applied Mechanics (ASME), 50(3):481 - 505.

Hellmich, C. (2005). Microelasticity of bone. In Dormieux, L. and Ulm, F.-J., editors, Applied Micromechanics of Porous Media, volume 480, pages 289 - 332. Springer, Wien - New York.

Hellmich, C., Kober, C., and Erdmann, B. (2008). Micromechanics-based conversion of CT data into anisotropic elasticity tensors, applied to FE simulations of a mandible. Annals of Biomedical Engineering, 36(1):108 - 122.

Hellmich, C. and Ulm, F.-J. (2001). Hydroxyapatite is uniformly concentrated in the extracollagenous ultrastructure of mineralized tissue. In Middleton, J., Shrive, N., and Jones, M., editors, Proceedings of the Fifth International Symposium on Computer Methods in Biomechanics and Biomedical Engineering, Rome, Italy.

Hellmich, C. and Ulm, F.-J. (2003). Average hydroxyapatite concentration is uniform in extracollageneous ultrastructure of mineralized tissue. Biomechanics and Modeling in Mechanobiology, 2:21 - 36.

Hellmich, C., Ulm, F.-J., and Dormieux, L. (2004). Can the diverse elastic properties of trabecular and cortical bone be attributed to only a few tissue-independent phase properties and their interactions? - arguments from a multiscale approach. Biomechanics and Modeling in Mechanobiology, 2:219 - 238.

Hershey, A. (1954). The elasticity of an isotropic aggregate of anisotropic cubic crystals. Journal of Applied Mechanics (ASME), 21:236 - 240.

Hill, R. (1963). Elastic properties of reinfored solids: some theoretical principles. Journal of the Mechanics and Physics of Solids, 11:357 - 362.

Hodge, A. and Petruska, J. (1963). Recent studies with the electron microscope on ordered aggregates of the tropocollagen molecule. In Ramachandran, G., editor, Aspects of Protein Structure - Proceedings of a Symposium held in Madras 14 - 18 January 1963 and organized by the University of Madras, India, pages 289 - 300. Academic Press, London and New York.

Hoffler, C. E., Moore, K. E., Kozloff, K., Zysset, P. K., and Goldstein, S. A. (2000). Age, gender, and bone lamellae elastic moduli. Journal of Orthopaedic Research, $18: 432-437$.

Höhling, H. (1969). Collagen mineralization in bone, dentine, cementum and cartilage. Naturwissenschaften, 56(9):466.

Jackson, D. and Hawkes, D. (1981). X-ray attenuation coefficients of elements and mixtures. Physics Letters, 70(3):169-233. 
Jilka, R., Weinstein, R., Bellido, T., Parfitt, A., and Manolagas, S. (1998). Osteoblast programmed cell death (apoptosis): Modulation by growth factors and cytokines. Journal of Bone and Mineral Research, 13(5):793-802.

Jowsey, J. (1960). Age changes in human bone. Clinical Orthopaedics, 17:210 - 218.

Karageorgiou, V. and Kaplan, D. (2005). Porosity of 3D biomaterial scaffolds and osteogenesis. Biomaterials, 26:5474-5491.

Katz, J. and Ukraincik, K. (1971). On the anisotropic elastic properties of hydroxyapatite. Journal of Biomechanics, 4:221 - 227.

Komlev, V., Mastrogiacomo, M., Pereira, R., Peyrin, F., Rustichelli, F., and Cancedda, R. (2010). Biodegradation of porous calcium phosphate scaffolds in an ectopic bone formation model studied by X-ray computed microtomography. European Cells and Materials, 19:136 - 146.

Landis, W., Hodgens, K., Song, M., Arena, J., Kiyonaga, S., Marko, M., Owen, C., and McEwen, B. (1996). Mineralization of collagen may occur on fibril surfaces: Evidence from conventional and high-voltage electron microscopy and threedimensional imaging. Journal of Structural Biology, 117:24 - 35.

Langer, R. and Vacanti, J. (1993). Tissue engineering. Science, 260(5110):920-926.

Laws, N. (1977). The determination of stress and strain concentrations at an ellipsoidal inclusion in an anisotropic material. Journal of Elasticity, 7:91 - 97.

Lees, S. (1987). Considerations regarding the structure of the mammalian mineralized osteoid from viewpoint of the generalized packing model. Connective Tissue Research, 16:281 - 303.

Lees, S. (2003). Mineralization of type I collagen. Biophysical Journal, 85:204 - 207.

Lees, S., Ahern, J., and Leonard, M. (1983). Parameters influencing the sonic velocity in compact calcified tissues of various species. Journal of the Acoustical Society of America, 74(1):28 - 33.

Lees, S., Bonar, L., and Mook, H. (1984a). A study of dense mineralized tissue by neutron diffraction. International Journal of Biological Macromolecules, 6:321 326.

Lees, S., Cleary, P., Heeley, J., and Gariepy, E. (1979a). Distribution of sonic plesio-velocity in a compact bone sample. Journal of Acoustic Society of America, 66(3):641-646.

Lees, S., Hanson, D., and Page, E. (1995). Some acoustical properties of the otic bones of a fin whale. Journal of the Acoustical Society of America, 99(4):2421 - 2427.

Lees, S., Hanson, D., Page, E., and Mook, H. (1994a). Comparison of dosagedependent effects of beta-aminopropionitrile, sodium fluoride, and hydrocortisone on selected physical properties of cortical bone. Journal of Bone and Mineral Research, 9(9): 1377 - 1389. 
Lees, S., Heeley, J., and Cleary, P. (1979b). A study of some properties of a sample of bovine cortical bone using ultrasound. Calcified Tissue International, 29:107 - 117.

Lees, S. and Page, E. (1992). A study of some properties of mineralized turkey leg tendon. Connective Tissue Research, 28:263 - 287.

Lees, S., Pineri, M., and Escoubes, M. (1984b). A generalized packing model for type I collagen. International Journal of Biological Macromolecules, 6:133 - 136.

Lees, S., Prostak, K., Ingle, V., and Kjoller, K. (1994b). The loci of mineral in turkey leg tendon as seen by atomic force microscope and electron microscopy. Calcified Tissue International, 55:180 - 189.

Lemaire, V., Tobin, F., Greller, L., Cho, C., and Suva, L. (2004). Modeling the interactions between osteoblast and osteoclast activities in bone remodeling. Journal of Theoretical Biology, 229:293 - 309.

McCarthy, R., Jeffcott, L., and McCartney, R. (1990). Ultrasound speed in equine cortical bone: effects of orientation, density, porosity and temperature. Journal of Biomechanics, 23(11):1139 - 1143.

Mori, T. and Tanaka, K. (1973). Average stress in matrix and average elastic energy of materials with misfitting inclusions. Acta Metallurgica, 21(5):571 - 574.

Noble, B. (2008). The osteocyte lineage. Archives of Biochemistry and Biophysics, 473:106 - 111 .

Parfitt, A. (1983). The physiologic and clinical significance of bone histomorphometric data. In Recker, R., editor, Histomorphometry, Techniques and Interpretation, pages 143-223. CRC Press Inc, Boca Raton, FL, USA.

Parry, D. and Craig, A. (1978). Collagen fibrils and elastic fibers in rat-tail tendon: an electron microscopic investigation. Biopolymers, 17:843 - 855 .

Perry, C. (2002). Biomaterials, synthetic synthesis, fabrication, and applications. In Meyer, R., editor, Encyclopedia of Physical Science and Technology, Volume 2, pages 173 - 191. Academic Press, San Diego, USA.

Pivonka, P., Zimak, J., Smith, D., Gardiner, B., Dunstan, C., Sims, N., Martin, T., and Mundy, G. (2010). Theoretical investigation of the role of the RANK-RANKL-OPG system in bone remodeling. Journal of Theoretical Biology, 262:306 - 316.

Pramatarova, L., Pecheva, E., Presker, R., Pham, M., Maitz, M., and Stutzmann, M. (2005). Hydroxyapatite growth induced by native extracellular matrix deposition on solid surfaces. European Cells and Materials, 9:9 - 12.

Rho, J., Zioupos, P., Currey, J., and Pharr, G. (2002). Microstructural elasticity and regional heterogeneity in human femoral bone of various ages examined by nanoindentation. Journal of Biomechanics, 35:189 - 198. 
Riedl, R. (1978). Order in Living Systems: A Systems Analysis of Evolution - Translated from "Die Ordnung des Lebendigen" by Parey, P. and tr. Jefferies, R.P.S. Wiley, New York.

Roeder, R. (2008). Hydroxyapatite-reinforced polymer biocomposites for synthetic bone substitutes. Journal of the Minerals, Metals and Materials Society, 60(3):38 45.

Roholl, P., Blauw, E., Zurcher, C., Dormans, J., and Theuns, H. (1994). Evidence for a diminished maturation of preosteoblasts into osteoblasts during aging in rats: an ultrastructural analysis. Journal of Bone and Mineral Research, 9(3):355 - 366.

Roschger, P., Gupta, H., Berzlanovich, A., Ittner, G., Dempster, D., Fratzl, P., Cosman, F., Parisien, M., Lindsay, R., Nieves, J., and Klaushofer, K. (2003). Constant mineralization density distribution in cancellous human bone. Bone, 32:316 - 323 .

Scheiner, S., Sinibaldi, R., Pichler, B., Komlev, V., Renghini, C., Vitale-Brovarone, C., Rustichelli, F., and Hellmich, C. (2009). Micromechanics of bone tissue-engineering scaffolds, based on resolution error-cleared computer tomography. Biomaterials, 30:2411-2419.

Teitelbaum, S. (2000). Bone resorption by osteoclasts. Science, 289:1504-1508.

Urist, M., DeLange, R., and Finerman, G. (1983). Bone cell differentiation and growth factors. Science, 220:680-686.

Verma, D., Katti, K., Katti, D., and Mohanty, B. (2008). Mechanical response and multilevel structure of biomimetic hydroxyapatite/polygalacturonic/chitosan nanocomposites. Materials Science and Engineering C, 28:399 - 405.

Wakashima, K. and Tsukamoto, H. (1991). Mean-field micromechanics model and its application to the analysis of thermomechanical behaviour of composite materials. Materials Science and Engineering: A, 146(1-2):291 - 316.

Weaver, J. (1966). The microscopic hardness of bone. Journal of Bone and Joint Surgery, 48:273-288.

Wergedal, J. and Baylink, D. (1974). Electron microprobe measurements of bone mineralization rate in vivo. American Journal of Physiology, 226(2):345-352.

Wolfram, U., Wilke, H.-J., and Zysset, P. (2010). Rehydration of vertebral trabecular bone: Influences on its anisotropy, its stiffness and the indentation work with a view to age, gender and vertebral level. Bone, 46:348 - 354 .

Zajac, A. and Discher, D. (2008). Cell differentiation through tissue elasticity-coupled, myosin-driven remodeling. Current Opinion in Cell Biology, 20(6):609-615.

Zaoui, A. (2002). Continuum micromechanics: survey. Journal of Engineering Mechanics, (ASCE), 128(8):808-816. 
Zhu, J.-X., Sasano, Y., Takahashi, I., Mizogushi, I., and Kagayama, M. (2001). Temporal and spatial gene expression of major bone extracellular matrix molecules during embryonic mandibular osteogenesis in rats. The Histochemical Journal, 33:25-35. 
- organic and mineral concentrations in bone tissue follow bilinear relationship

- micromechanics provides experimentally validated mass density-elasticity relationships

- this opens new routes for evaluation of Computer Tomographic data 